\title{
Large-eddy simulation of conditionally neutral boundary layers: a mesh resolution sensitivity study
}

Berg, Jacob; Patton, Edward G.; Sullivan, Peter P.

Published in:

Journal of the Atmospheric Sciences

Link to article, DOI:

10.1175/jas-d-19-0252.1

Publication date:

2020

Document Version

Publisher's PDF, also known as Version of record

Link back to DTU Orbit

Citation (APA):

Berg, J., Patton, E. G., \& Sullivan, P. P. (2020). Large-eddy simulation of conditionally neutral boundary layers: a mesh resolution sensitivity study. Journal of the Atmospheric Sciences, 77, 1969-1991.

https://doi.org/10.1175/jas-d-19-0252.1

\section{General rights}

Copyright and moral rights for the publications made accessible in the public portal are retained by the authors and/or other copyright owners and it is a condition of accessing publications that users recognise and abide by the legal requirements associated with these rights.

- Users may download and print one copy of any publication from the public portal for the purpose of private study or research.

- You may not further distribute the material or use it for any profit-making activity or commercial gain

- You may freely distribute the URL identifying the publication in the public portal 


\title{
Large-Eddy Simulation of Conditionally Neutral Boundary Layers: A Mesh Resolution Sensitivity Study
}

\author{
JACOB BERG \\ Department of Wind Energy, Ris $\phi$ Campus, Technical University of Denmark, Roskilde, Denmark \\ Edward G. Patton AND Peter P. Sullivan \\ National Center for Atmospheric Research, Boulder, Colorado
}

(Manuscript received 16 September 2019, in final form 17 March 2020)

\begin{abstract}
Large-eddy simulation (LES) is used to model turbulent winds in a nominally neutral atmospheric boundary layer at varying mesh resolutions. The boundary layer is driven by wind shear with zero surface heat flux and is capped by a stable inversion. Because of entrainment the boundary layer is in a weakly stably stratified regime. The simulations use meshes varying from $128^{2} \times 64$ to $1024^{2} \times 512$ grid points in a fixed computational domain of size $(2560,2560,896) \mathrm{m}$. The subgrid-scale (SGS) parameterizations used in the LES vary with the mesh spacing. Low-order statistics, spectra, and structure functions are compared on the different meshes and are used to assess grid convergence in the simulations. As expected, grid convergence is primarily achieved in the middle of the boundary layer where there is scale separation between the energycontaining and dissipative eddies. Near the surface second-order statistics do not converge on the meshes studied. The analysis also highlights differences between one-dimensional and two-dimensional velocity spectra; differences are attributed to sampling errors associated with aligning the horizontal coordinates with the vertically veering mean wind direction. Higher-order structure functions reveal non-Gaussian statistics on all scales, but are highly dependent on the mesh resolution. A generalized logarithmic law and a $k^{-1}$ spectral scaling regime are identified with mesh-dependent parameters in agreement with previously published results.
\end{abstract}

\section{Introduction}

Large-eddy simulation (LES) has become an essential tool for studying a wide range of societal pertinent atmospheric boundary layer (ABL) applications (e.g., wind energy meteorology; Calaf et al. 2010; Abkar and Porté-Agel 2013; Churchfield et al. 2012; Sørensen et al. 2015; Allaerts and Meyers 2015) where accurate simulation and representation of near-surface processes are needed. Hence the community needs to thoroughly investigate the numerous assumptions, components, and choices underpinning LES.

Although the fundamentals underpinning LES remain similar to their original formulation introduced numerous years ago (Lilly 1967; Deardorff 1970, 1972), recent advances in high-performance computing allows for larger domains and higher resolutions such that LES can now simulate a sufficiently wide inertial range to

Corresponding author: Jacob Berg, jacob.berg.joergensen@gmail.com provide important insight into turbulence beyond second-order statistics (Stevens et al. 2014). Since the physical subgrid-scale model (SGS) used to close the equations depends on resolution, mesh size is a crucial factor. Increasing the number of grid points for simulations using similar domain lengths implies that finer turbulent structures are resolved. In the atmospheric surface layer (i.e., the portion of the ABL directly influenced by the underlying ground surface), the characteristic length scale of turbulence grows as $\kappa z$, where $\kappa \sim 0.4$ is the von Kármán constant and $z$ is the distance from the surface. Thus, close to the surface high resolution is needed. The inherent problem of resolving the surface layer and capturing the logarithmic law describing the mean wind speed is well described by Brasseur and Wei (2010) and will also be discussed later in this paper.

A target of the present work is to examine the impact of mesh resolution $\Delta$ on neutral ABL turbulence generated by LES. In typical LES applications, the SGS 
model utilizes a turbulent eddy viscosity prescription $\nu_{t} \sim \sqrt{e} \Delta$ where $e$ is the SGS energy and $\ell$ is a length scale; the SGS parameterization is made scale aware by choosing $\ell \sim \Delta$. Thus, variations in mesh spacing or equivalently turbulent eddy viscosity change the large-eddy Reynolds number $\operatorname{Re}_{\ell}$ of the simulation (see section $4 \mathrm{a}$ ). The impact of Reynolds number on turbulence is a traditional focus of direct numerical simulation (DNS) studies (e.g., Moin and Mahesh 1998), but its impact in atmospheric LES is rarely studied (e.g., Sullivan and Patton 2011; Bou-Zeid 2015).

In general, studying the effect of mesh resolution across the entire $\mathrm{ABL}$ is also relevant for applications where realistic turbulence profiles are needed (e.g., wind turbines operate in the lowest couple of hundred meters of the ABL and the turbulence is responsible for the loading and hence fatigue on the turbines). When deciding where to position a wind turbine (i.e., choosing the correct turbine class to match the local site conditions) (International Electrotechnical Commission 2005), any uncertainty in predicting turbulence levels will manifest itself as an increased uncertainty of the turbine's life expectancy and operation window.

The ABL can be naturally divided into three main canonical classes: the 1) neutral, 2) convective (or unstable), and 3) stable boundary layer, which can largely be characterized by zero, positive, and negative surface heat fluxes, respectively. The effect of mesh resolution on turbulence simulated by large-eddy simulation in the latter two ABL classes has been carefully studied by Sullivan and Patton (2011, convective) and Sullivan et al. (2016, stable) with the same pseudospectral model utilized here. Both of those studies found that second-order turbulence statistics can be strongly affected by mesh size. In those studies, the ABL was also capped by potential temperature inversion creating a region through which warm air entrains from above by eddies of decreasing size as the turbulence interacts with the overlying stratification. Since increasing resolution alters the range of turbulent structures resolved, high resolution also potentially impacts the region surrounding the capping inversion and hence the ability to properly simulate ABL growth. We therefore use a similar setup to the previous two aforementioned studies but examine the asymptotic situation of negligible heat flux at the surface (i.e., the neutral boundary layer in which production of turbulent kinetic energy primarily occurs through shear but where heat entrained at the top of the ABL ensures weak stable stratification throughout the ABL). This type of canonical boundary layer has been called an inversion capped neutral boundary layer or a conditionally neutral boundary layer in contrast to the Neutral Ekman boundary layer where temperature effects are completely absent. Neutral Ekman boundary layers were also recently studied with the same LES model as in our study (Jiang et al. 2018), and it was found that increased horizontal grid resolution has a profound effect on the size of the logarithmic layer near the ground by thinning the layer closest to the surface where the most energetic eddies are underresolved by the LES.

Several studies have focused on LES of conditionally neutral boundary layers: Lin et al. (1996) focuses mainly on the momentum flux and vorticity balance, Pedersen et al. (2014) showed the development of boundary layers from looking at the terms in the turbulent kinetic energy (TKE) equation, and Otte and Wyngaard (2001) primarily focused on the interfacial layer around the capping inversion, while Moeng and Sullivan (1994) compared the overall structure in relation to stable and convective boundary layers. Furthermore, wind farm studies have been presented by Abkar and Porté-Agel (2013) and Allaerts and Meyers $(2015,2017)$ focusing on how the strength of the inversion affects wind turbine performance within farms as a result of altered momentum fluxes in the wake regions. Pollard et al. (1973) and Zilitinkevich et al. (2007) showed that the height of the boundary layer also depend on the Brunt-Väisälä frequency, thus adding another time scale to the problem in addition to that introduced by the Coriolis force. Common for all the studies just mentioned is that all the simulations discussed use fairly coarse resolution compared to those examined by Sullivan and Patton (2011) and Sullivan et al. (2016). Pedersen et al. (2014) claimed to observe mesh independence when it comes to capturing the log law at the surface and when estimating the boundary layer growth rate $w_{e}$ (in the absence of subsidence); but we will show mesh dependencies for statistics even down to second order for simulations using similar domain sizes but resolved by up to $1024^{2} \times 512$ grid points on a domain with physical size $(2560 \mathrm{~m})^{2} \times$ $896 \mathrm{~m}$. It should also be noted that Pan and Chamecki (2016) found a resolution dependence in the logarithmic layer of a canopy ABL when looking at non-Gaussian effects at the smallest scales through velocity structure functions.

The paper is organized as follows: section 2 presents a description of the LES and its configuration. Section 3 provides and overview of the suite of LES simulations studied including the ABL development and sampling/averaging procedure considerations. Section 4 contains the analysis and discussions, and section 5 provides a summary of the findings.

\section{LES equations}

We use the National Center for Atmospheric Research (NCAR) pseudospectral LES code (Sullivan and Patton 
TABLE 1. Overview of simulations.

\begin{tabular}{ccccccc}
\hline \hline Name & Grid points & $\left(L_{x}, L_{y}, L_{z}\right)(\mathrm{m})$ & $(\Delta x, \Delta y, \Delta z)(\mathrm{m})$ & $\Delta_{f}(\mathrm{~m})$ & $\Delta t_{\mathrm{avg}}(\mathrm{s})$ & $T(\mathrm{~s})$ \\
\hline A & $128^{2} \times 64$ & $(2560,2560,896)$ & $(20.0,20.0,14.0)$ & 23.3 & 3.86 & $1.2 \times 10^{5}$ \\
B & $256^{2} \times 128$ & $(2560,2560,896)$ & $(10.0,10.0,7.0)$ & 11.6 & 1.85 \\
C & $512^{2} \times 256$ & $(2560,2560,896)$ & $(5.0,5.0,3.5)$ & 5.8 & 0.88 & $2.3 \times 10^{5}$ \\
D & $1024^{2} \times 512$ & $(2560,2560,896)$ & $(2.5,2.5,1.75)$ & 2.9 & 0.43 & $1.1 \times 10^{5}$ \\
\hline
\end{tabular}

2011; Sullivan et al. 2016), mimicking a dry atmospheric boundary layer (ABL) under the Boussinesq approximation over a flat lower boundary with constant roughness length $z_{0}$. The origin of the model goes back to the original work of Deardorff (1970) with the novel modifications introduced by Moeng (1984) and Moeng and Wyngaard (1988).

The LES equations are formulated in a right handed Cartesian coordinate system, $\mathbf{x}=\left(x_{1}, x_{2}, x_{3}\right)=(x, y, z)$, for the spatial filtered variables $\tilde{u}_{i}(\mathbf{x}, t), \tilde{\theta}(\mathbf{x}, t), \tilde{\pi}(\mathbf{x}, t)$, and $e(\mathbf{x}, t)$, which are the velocity, potential temperature, modified hydrodynamic pressure (normalized by a reference density), and subgrid-scale (SGS) kinetic energy, respectively:

$$
\begin{aligned}
\partial_{t} \tilde{u}_{i}+\tilde{u}_{i} \partial_{j} \tilde{u}_{j} & =-\partial_{i} \tilde{\pi}+\varepsilon_{i j 3} f\left(\tilde{u}_{i}-U_{i}^{G}\right)+\beta \tilde{\theta} \delta_{i 3}-\partial_{j} \tau_{i j} \\
\partial_{i} \tilde{u}_{i} & =0 \\
\partial_{t} \tilde{\theta}+\tilde{u}_{i} \partial_{i} \tilde{\theta} & =-\partial_{i} \tau_{i \theta} \\
\partial_{t} e+\tilde{u}_{i} \partial_{i} e & =\mathscr{P}+\mathscr{B}+\mathscr{D}-\mathscr{E}
\end{aligned}
$$

The incompressibility condition listed in Eq. (2) leads to a Poisson equation for the pressure variable $\tilde{\pi}$. The advection term in Eq. (1) is written in rotational form, and the vertical momentum equation $(i=$ 3 ) is solved only for the part deviating from hydrostatic balance and the horizontal mean (i.e., $\left\langle\tilde{u}_{3}\right\rangle=0$ at all heights) (Moeng 1984). The model is forced by a geostrophic relationship with a constant height independent geostrophic velocity $U_{i}^{G}$ and Coriolis parameter $f$ (i.e., mimicking barotropic conditions). The buoyancy parameter, $\beta=g / \theta_{0}$, is held constant with gravitational acceleration $g=9.81 \mathrm{~m} \mathrm{~s}^{-2}$ and reference potential temperature $\theta_{0}=290 \mathrm{~K}$. Where appropriate we will use explicit variable naming; $(u, v$, $w)$ instead of the more compact Einstein notation $\left(u_{1}\right.$, $u_{2}, u_{3}$ ) for the velocity vector.

We use Deardorff's (1980) SGS model with a stabilitycorrected length scale. Details of the right-hand-side terms in Eq. (4) representing shear production $\mathscr{P}$, buoyancy $\mathscr{B}$, diffusion $\mathscr{D}$, and dissipation $\mathscr{E}$ as well as the SGS terms in Eqs. (1)-(4):

$$
\begin{aligned}
\tau_{i j} & =\widetilde{u_{i} u_{j}}-\tilde{u}_{i} \tilde{u}_{j}-\frac{\delta_{i j}}{3}\left(\widetilde{u_{k} u_{k}}-\tilde{u}_{k} \tilde{u}_{k}\right), \\
\tau_{i \theta} & =\widetilde{u_{i} \theta}-\tilde{u}_{i} \tilde{\theta}, \\
e & =\frac{1}{2}\left(\widetilde{u_{i} u_{i}}-\tilde{u}_{i} \tilde{u}_{i}\right),
\end{aligned}
$$

can be found in Deardorff (1980), Moeng (1984), and Moeng and Wyngaard (1988). Near the surface we use the two-part SGS eddy-viscosity model extension that explicitly accounts for the near-surface mean flow (Sullivan et al. 1994). For all simulations discussed, the scale at which the equations are filtered falls at scales at least decades larger $O(1-10)$ m than the Kolmogorov microscale $O(1) \mathrm{mm}$; therefore, the viscous terms that would otherwise appear in Eq. (1) have been neglected. As in both Sullivan and Patton (2011) and Sullivan et al. (2016) the focus in this paper is on the dependence of mesh resolution given a specific SGS model. Results may therefore differ when applying SGS models of other types (such as the different dynamic Smagorinsky

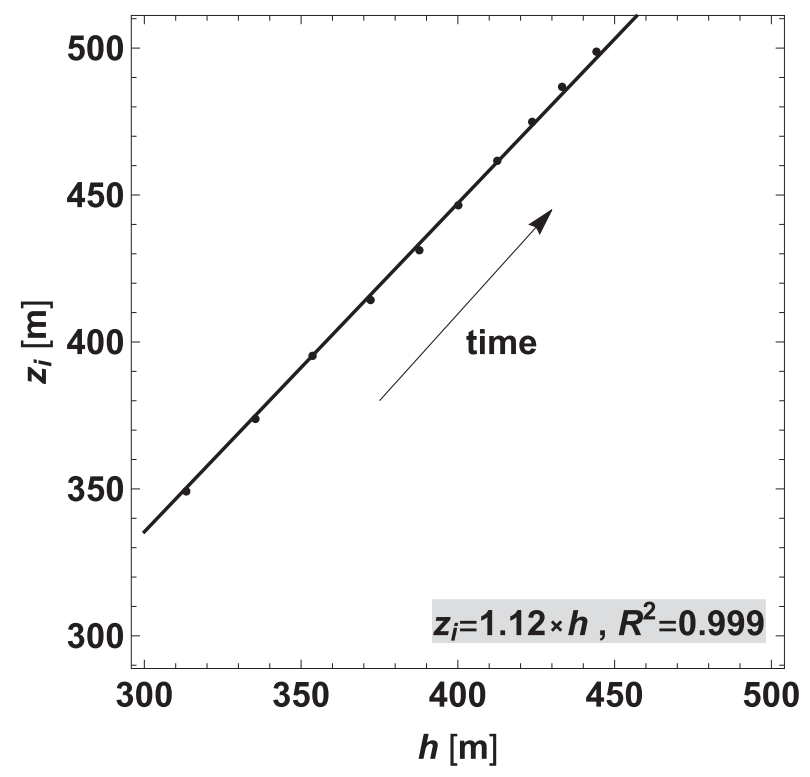

FIG. 1. The relationship between $z_{i}$ and $h$ for simulation times between $35 T_{E}$ and $-135 T_{E}$ in steps of $10 T_{E}$ from run $\mathrm{B}$. The fit is forced to have a zero intercept. 

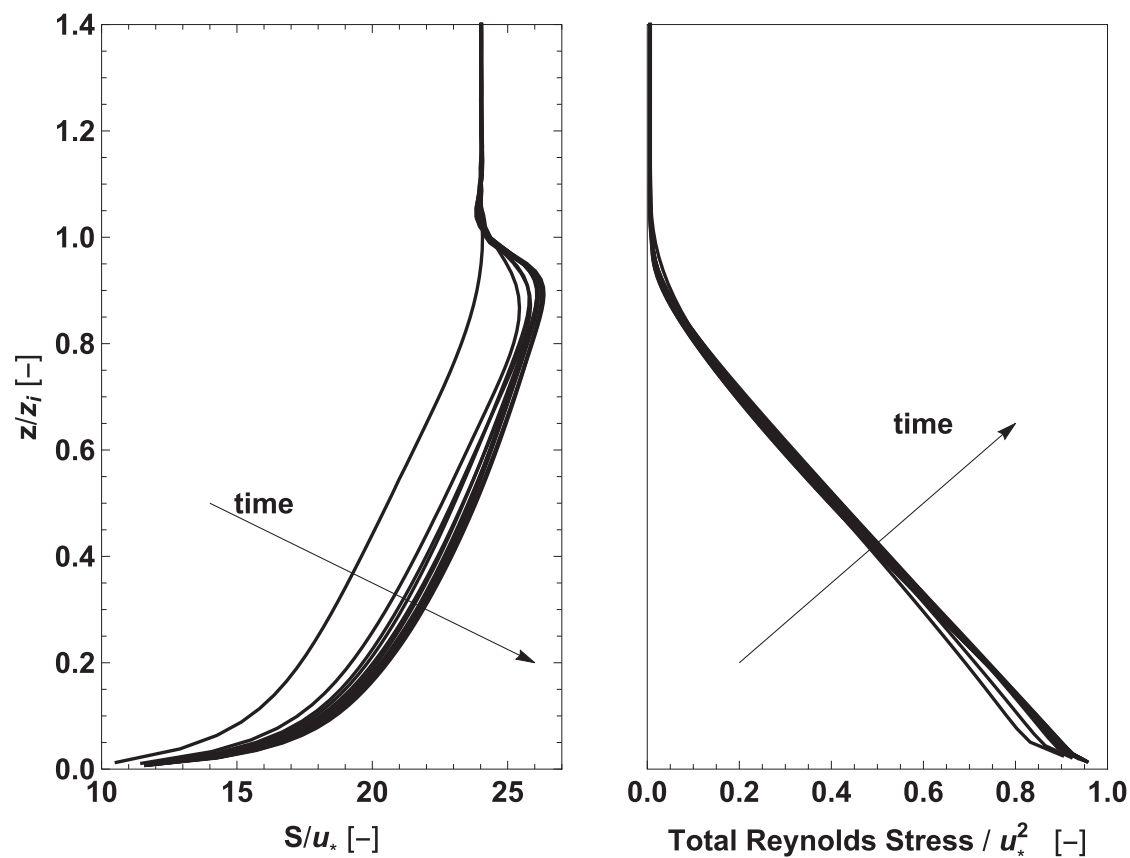

FIG. 2. Profiles of (left) wind speed $S$ and (right) normalized total Reynolds stress $\left(\left\langle\tilde{u}^{\prime} \tilde{w}^{\prime}+\tau_{13}\right\rangle^{2}+\left\langle\tilde{v}^{\prime} \tilde{w}^{\prime}+\tau_{23}\right\rangle^{2}\right)^{1 / 2}$. Profiles are plotted averaging intervals from $5 T_{E}$ to $135 T_{E}$ in steps of $10 T_{E}$. Data are presented for run B.

models introduced and discussed in Porté-Agel et al. 2000; Meneveau and Katz 2000; Bou-Zeid et al. 2005, 2008).

We apply rough wall boundary conditions at the lower bottom "surface" through specification of MoninObukhov similarity functions (Moeng 1984; Moeng and Sullivan 1994). At the upper boundary a radiation condition is applied (Klemp and Durran 1983). We use periodic boundary conditions at all lateral walls. Broad details regarding code parallelization and use of fast Fourier transforms to solve the Poisson equation for the nonlocal pressure can be found in Sullivan and Patton (2011).

Time is advanced through a third-order Runge-Kutta scheme, where the time step is dynamically calculated each iteration based upon a constant Courant-Friedrichs-Lewy (CFL) number of 0.5. We take advantage of the Galilean invariance of the governing equations and hence move the mesh with a speed equal to half the geostrophic speed $U^{G}$, which permits approximately a factor-of-2 larger time step.

\section{Design of LES experiments}

a. Grid mesh

The focus is on mesh dependence of shear driven boundary layers under weakly stable stratification, hence a series of simulations have been conducted with varying mesh resolution from $128^{2} \times 64$ to $1024^{2} \times 512$ (Table 1). All simulations use the same domain size $\left(L_{x}\right.$, $\left.L_{y}, L_{z}\right)=(2560,2560,896) \mathrm{m}$; however, the simulation duration $T$ differs slightly across simulations. We denote the average time step in the simulations with $\Delta t_{\text {avg. }}$. As is common practice in pseudospectral codes in order to avoid aliasing effects, the top one-third of wavenumbers are ignored (Orszag 1971), meaning that the spatial resolution [i.e., the effective LES filter width is $\Delta_{f}=[(3 / 2) \Delta x$, $\left.(3 / 2) \Delta y, \Delta z]^{1 / 3}\right]$. Due to the doubling of resolution at each simulation level the aspect ratio, $\Delta x / \Delta z=\Delta y / \Delta z$, is held constant in our study. Brasseur and Wei (2010) discuss possible implications of grid aspect ratio for the "overshoot" problem in the logarithmic layer, and

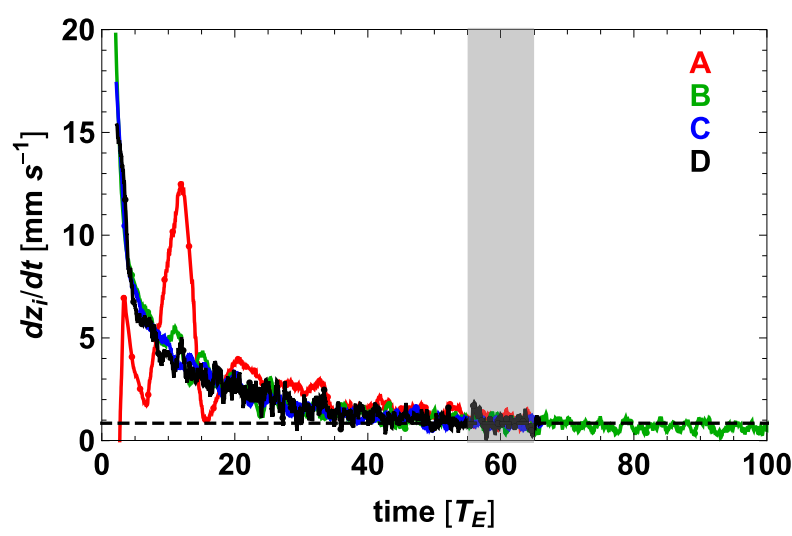

FIG. 3. Growth of $z_{i}$ as a function of simulation time in units of the eddy-turn over time $T_{E}$. The dashed horizontal line indicates $0.85 \mathrm{~mm} \mathrm{~s}^{-1}$. The colors refer to the four simulations A-D. 


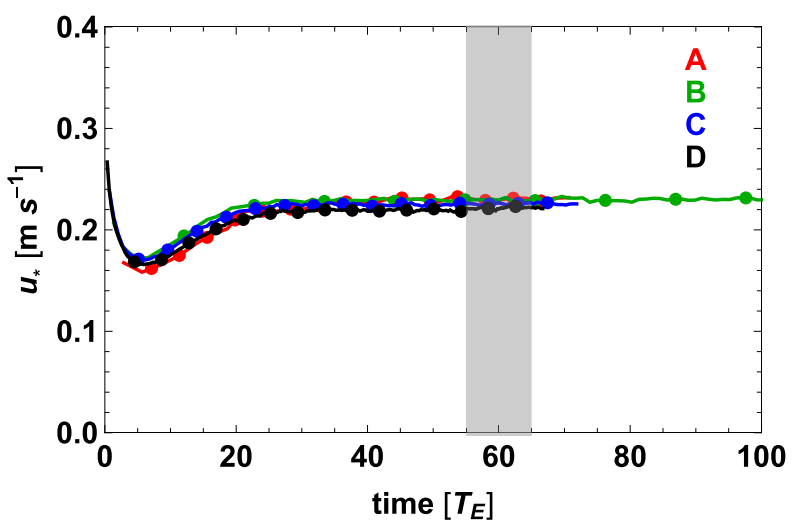

FIG. 4. Friction velocity of $u_{\star}$ as a function of simulation time in units of the eddy-turnover time $T_{E}$. The colors refer to the four simulations A-D.

Ercolani et al. (2017) finds an optimal value of 4 (it is $\sim 1.4$ in our study and thus closer to the isotropy assumption in the SGS model), although it is not clear whether that result applies to incompressible pseudospectral solvers like the NCAR LES code.

\section{b. Imposed parameters}

The externally imposed parameters defining the canonical $\mathrm{ABL}$ are held constant for all runs, these include: surface heat flux $Q_{0}=0 \mathrm{~K} \mathrm{~m} \mathrm{~s}^{-1}$, geostrophic velocity $\left(U^{G}, V^{G}\right)=(5,0) \mathrm{m} \mathrm{s}^{-1}$, Coriolis parameter $f=$ $10^{-4} \mathrm{~s}^{-1}$, and surface roughness length $z_{0}=0.05 \mathrm{~m}$. All simulations are initialized with constant potential temperature gradient, $\partial \tilde{\theta}_{f} / \partial z=0.003 \mathrm{~K} \mathrm{~m}^{-1}$, throughout the domain. The subscript $f$ refers to the "free" atmosphere (i.e., the nonturbulent atmosphere into which the boundary layer develops). As time advances, boundary layer growth occurs as turbulent kinetic energy is produced by shear production at the surface and entrainment of potential temperature at the top. The stable stratification capping the ABL from above is characterized by a BruntVäisälä frequency, $N^{2}=\beta \partial \tilde{\theta}_{f} / \partial z=10^{-4} \mathrm{~s}^{-2}$. The coordinate system is aligned with $i=1$ in the direction of the geostrophic wind, $U^{G}$, which has consequences for onedimensional spectral properties (see section 4c).

The velocity fields are initialized with the geostrophic wind profile. Incompressible velocity and temperature fluctuations initiate turbulence in the lowest $50 \mathrm{~m}$. Following Beare et al. (2006), horizontally homogeneous vertical profile of SGS kinetic energy is initialized in the lowest $250 \mathrm{~m}$ as $e(z)=0.4(1-z / 250)$.

\section{c. Averaging procedures}

Due to horizontal homogeneity we approximate ensemble averaging (denoted with angle brackets) with horizontal averaging, which then becomes only a function of the vertical direction $z$. We denote the local fluctuations in a variable $\chi(x, y, z, t)$ as departures from the horizontal average according to

$$
\tilde{\chi}^{\prime}(x, y, z, t)=\tilde{\chi}(x, y, z, t)-\langle\tilde{\chi}\rangle(z, t),
$$

where the time dependence has been kept in order to emphasize the inherent statistical nonstationarity of the flow. We use the longest duration simulation (run B) to identify a pseudostationary time period for comparison of the different mesh resolution simulations.

At every time step, we estimate the boundary layer height using two different methods: 1) the "maximum gradient method" applied to potential temperature (Sullivan et al. 1998) leading to $z_{i}$, and 2) finding the height $h$ at which the square root of the total Reynolds stress $\left(\left\langle\tilde{u}^{\prime} \tilde{w}^{\prime}+\tau_{13}\right\rangle^{2}+\left\langle\tilde{v}^{\prime} \tilde{w}^{\prime}+\tau_{23}\right\rangle^{2}\right)^{1 / 4}$ falls to $5 \%$ of its surface value $u_{\star}$ (Kosović and Curry 2000). We evalue $u_{\star}$ at $z=\Delta z / 2$ (i.e., in the first grid point from the resolved horizontal velocities $\tilde{u}$ and $\tilde{v}$ through the logarithmic law). Figure 1 compares $z_{i}$ and $h$ for times larger than $35 T_{E}$ for run B (i.e., at times after the initial spinup). We observe a linear relationship with a ratio, $z_{i} / h=1.12$ Since absolute numbers are not relevant, from here onward we will refer to $z_{i}$ as our ABL height estimate.

To compare the four simulations, we define a largeeddy turn-over time scale as $T_{E}=z_{i} / u_{\star}$. Figure 2 shows profiles of mean wind speed, $S=\left(\langle\tilde{u}\rangle^{2}+\langle\tilde{v}\rangle^{2}\right)^{1 / 2}$, and the total Reynolds Stress as a function of time, where we present profiles for the longest simulation, run B, averaged over intervals ranging from $\{5-15\} T_{E}$ to $\{125-135\}$ $T_{E}$. In both panels we see that the profiles each approach a limiting profile as time advances. At $\{55-65\} T_{E}$ we conclude that the profile shapes are similar to the limiting shapes and that a pseudo-stationary state has been reached.

Figure 3 shows the growth rate (or alternatively the entrainment rate), $w_{e}=\partial z_{i} / \partial t$, as a function of the eddy

TABLE 2. Bulk parameters.

\begin{tabular}{ccccccrrr}
\hline \hline Name & $h(\mathrm{~m})$ & $z_{i}(\mathrm{~m})$ & $z_{i} / h(-)$ & $u_{\star}\left(\mathrm{m} \mathrm{s}^{-1}\right)$ & $z_{i}(|f| N)^{1 / 2} u_{\star}^{-1}(-)$ & $T_{E}(\mathrm{~s})$ & $z_{i} / \Delta_{f}(-)$ & $\mathrm{Re}_{\ell}(-)$ \\
\hline $\mathrm{A}$ & 348 & 399 & 1.15 & 0.230 & 1.74 & 1733 & 17.1 \\
$\mathrm{~B}$ & 353 & 395 & 1.12 & 0.229 & 1.73 & 1722 & 34.1 \\
$\mathrm{C}$ & 338 & 372 & 1.10 & 0.225 & 1.65 & 1648 & 64.1 \\
$\mathrm{D}$ & 321 & 350 & 1.09 & 0.221 & 1.59 & 1579 & 120.1 & 2012 \\
\hline
\end{tabular}




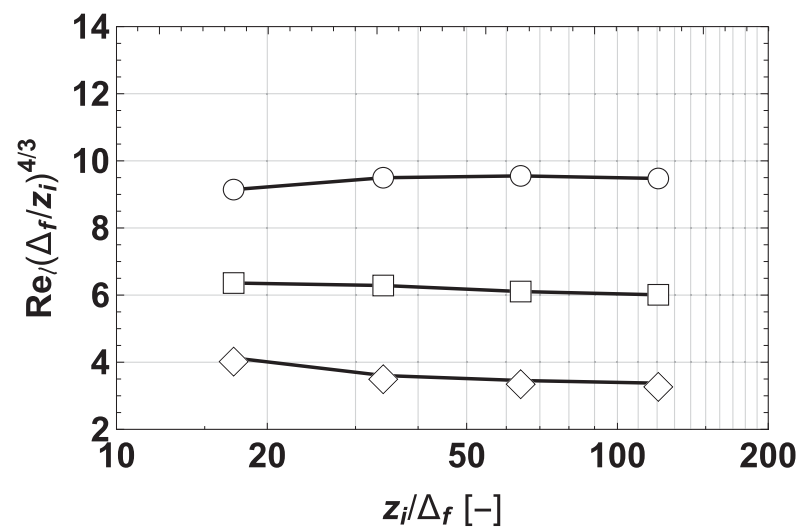

FIG. 5. $\operatorname{Re}_{\ell}$ multiplied with $\left(\Delta_{f} / z_{i}\right)^{4 / 3}$ as a function of $z_{i} / \Delta_{f}$ for the three heights $z / z_{i}=0.1$ (diamonds), 0.5 (squares), and 0.9 (circles).

turnover time $T_{E}$. At $\{55-65\} T_{E}$, the growth rate reaches a constant value of $w_{e} \sim 0.85 \mathrm{~m} \mathrm{~s}^{-1}$ and the variation in $w_{e}$ among the four simulations is less than $5 \%$. This time interval $\left(\{55-65\} T_{E}\right)$ can therefore be assumed quasi stationary and is used from here forward as our averaging interval. Figure 4 shows that the friction velocity $u_{\star}$ also converges by $\{55-65\} T_{E} ; u_{\star}$ actually converges much earlier, at around $30 T_{E}$, since $u_{\star}$ is by definition governed by surface processes in contrast to $z_{i}$.

\section{Results}

This section presents mesh resolution sensitivity results. Bulk parameters from the simulations are calculated using averages over the interval $\{55-65\} T_{E}$. The numbers are presented in Table 2.

\section{a. Large-eddy Reynolds number and SGS dissipation}

In Sullivan and Patton (2011) on convective boundary layers the ratio $z_{i} / \Delta_{f}$ was used as a measure of the ABL resolution as it represents the separation between the largest possible energy-containing eddy in the flow and the scales of motion nearest to the LES cutoff. In Fig. 1 we have shown that in our conditional neutral boundary layer $z_{i} \sim 1.12 h$ for the B run. Since the ratio $z_{i} / h$, as can be seen in Table 2, only changes slightly with mesh size, we use the ratio $z_{i} / \Delta_{f}$ to express $\mathrm{ABL}$ resolution. With $z_{i} \in\{350$ $399\} \mathrm{m}$ across the four simulations, we furthermore find that the domain size is large enough compared to the ABL height; the "rule of thumb" says that $L_{x} / z_{i}>5, L_{y} / z_{i}>5$, and $L_{z} / z_{i}>2$ needs to be satisfied (reference unknown).

The resolved turbulence in the high-Reynolds-number LES should be independent of processes taken place on scales below the filter scale $\Delta_{f}$. To state that a given mesh is fine enough and that the simulation has is independent of the SGS viscosity is equivalent to stating that the large-eddy Reynolds number is a function of

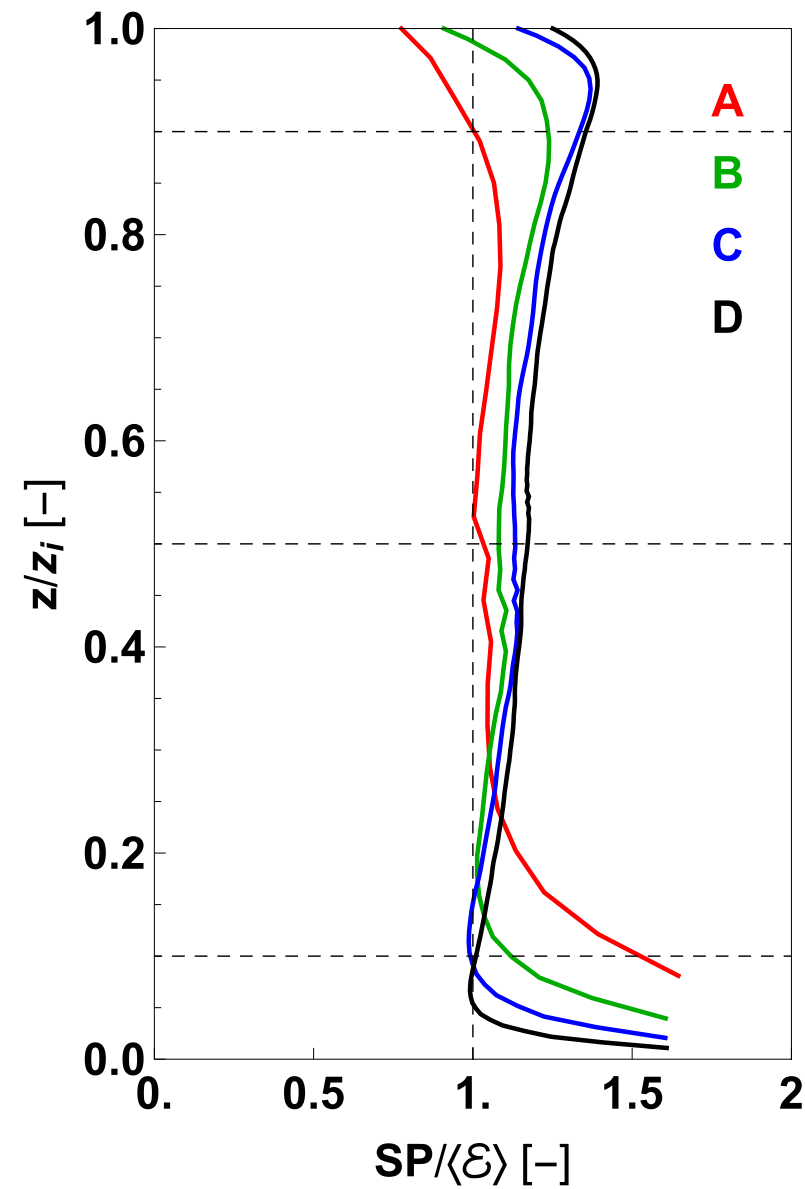

FIG. 6. Ratio between resolved shear production $\mathrm{SP}=$ $-\left(\left\langle\tilde{u}^{\prime} \tilde{w}^{\prime}\right\rangle \partial\langle\tilde{u}\rangle / \partial z+\left\langle\tilde{v}^{\prime} \tilde{w}^{\prime}\right\rangle \partial\langle\tilde{\boldsymbol{v}}\rangle / \partial z\right)$ and dissipation $\langle\mathscr{E}\rangle$ as a function of normalized height $z / z_{i}$.

the effective resolution alone. The effective resolution is here defined as the ratio between the largest and smallest scales resolved in the simulation $z_{i} / \Delta_{f}$. We define the large-eddy Reynolds number:

$$
\operatorname{Re}_{\ell}=\frac{u \ell}{\nu_{t}} \equiv \frac{u_{\star} z_{i}}{C_{k} \Delta_{f} e^{1 / 2}}
$$

where in contrast to Moeng and Wyngaard (1988) and Sullivan and Patton (2011) the friction velocity $u_{\star}$ is the characteristic velocity scale since turbulence in our study is produced by shear. In Eq. (9) we use a definition of SGS viscosity consistent with that used in the LES code; $C_{k}=0.1$. Pollard et al. (1973) propose $\ell=c_{c n} u_{\star}(|f| N)^{-1 / 2}$ from studies with a simple model of the upper ocean. From ABL LESs Zilitinkevich et al. (2007) find $c_{c n}=1.36$ using same definition of $h$ as used in our study. The spinup time in their LES is, however, different compared to our study, and hence a different numerical value should be expected, due to the 

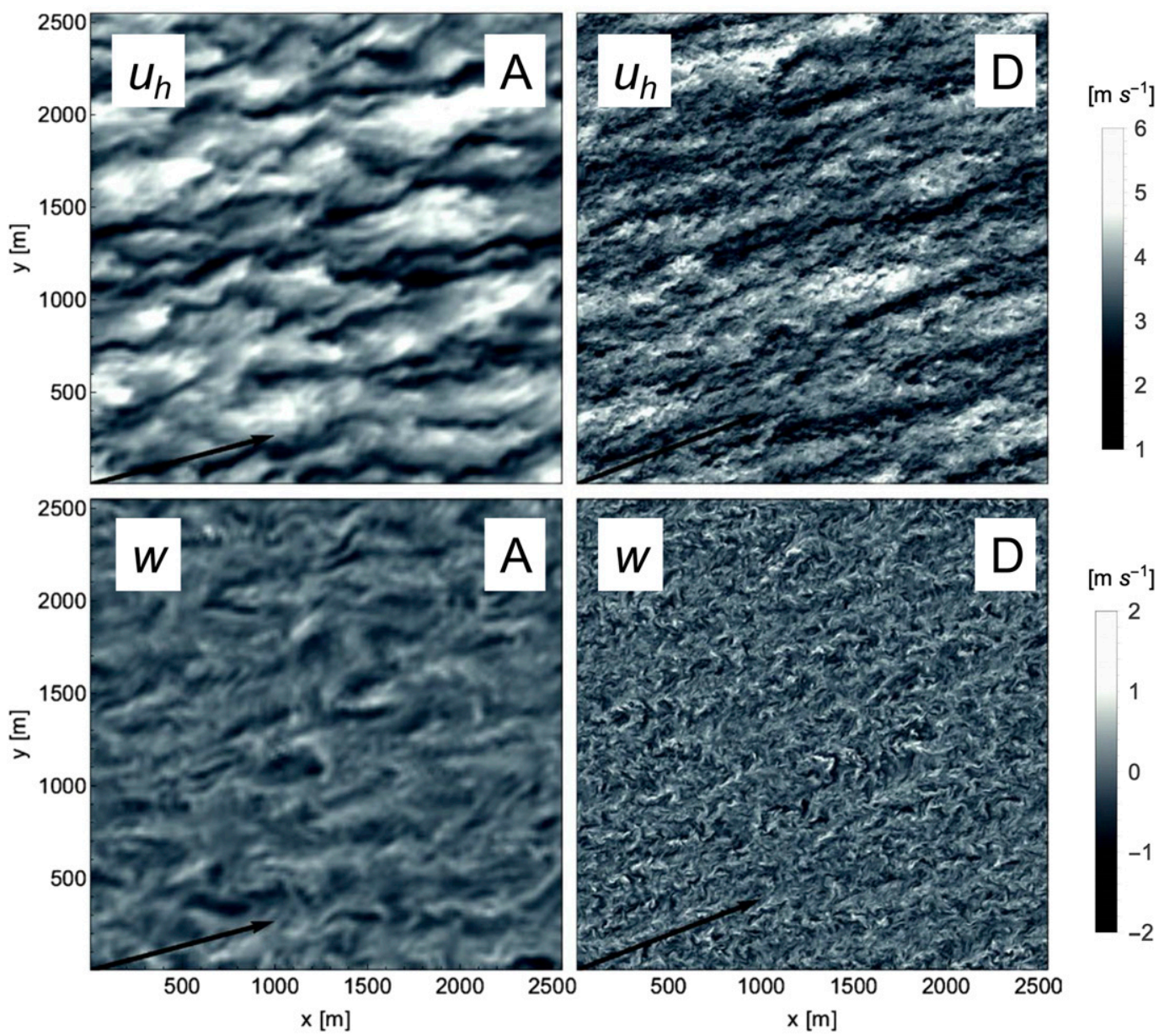

FIG. 7. Snapshots of the (top) streamwise velocity $u_{h}$ and (bottom) vertical velocity $w$ at $z / z_{i}=0.1$ for (left) simulation A and (right) simulation D. Black arrows in the lower-left corner of each panel represent the mean wind direction.

inherent lack of stationarity in the problem. We adopt $z_{i}=c_{c n^{\prime}} u_{\star}(|f| N)^{-1 / 2}$, and find values of $c_{c n^{\prime}}$ as provided in Table 2; most pronounced is a small dependence on mesh size. Together with the SGS model (Lilly 1967; Moeng and Wyngaard 1988) (here without the stability correction-see section $4 \mathrm{~b}), \mathscr{E}=C_{\mathscr{\delta}} e^{3 / 2} / \Delta_{f}$, and the SGS identity of constants $\left(c_{s}=C_{k}^{3 / 4} c_{\mathscr{E}}^{-1 / 4}\right.$ with $\left.c_{s}=0.18\right)$ we can also express the large-eddy Reynolds number as

$$
\operatorname{Re}_{\ell}=\left(\frac{z_{i}}{c_{s} \Delta_{f}}\right)^{4 / 3}\left[\frac{u_{\star}^{2}(|f| N)^{1 / 2}}{c_{c n^{\prime}} \mathscr{E}}\right]^{1 / 3} .
$$

We thus recover the $\left(z_{i} / \Delta_{f}\right)^{4 / 3}$ dependence also found in Sullivan and Patton (2011) and in direct numerical simulation (Pope 2000). Since the product of all the other parameters in the second parentheses of Eq. (10) are mesh independent $\left(u_{\star}^{2} / c_{c n^{\prime}}=0.030 \mathrm{~m}^{2} \mathrm{~s}^{-2}\right.$ in all four simulations), $\mathscr{E}$ needs to be constant in the inertial subrange (assuming that $\Delta_{f}$ is in the inertial subrange).
In Fig. 5 we plot $\mathrm{Re}_{\ell}$ multiplied with $\left(\Delta_{f} / z_{i}\right)^{4 / 3}$ for the three heights: $z / z_{i}=0.1, z / z_{i}=0.5$, and $z / z_{i}=0.9$. At $z / z_{i}=0.9$. we observe mesh independence going from simulation C to D. Closer near the surface where the dissipation rates $\mathscr{E}$ are much higher, mesh independence is still not achieved. In Sullivan and Patton (2011) mesh independence through a similar analysis was reported from $z_{i} / \Delta_{f}>60$ in a convective boundary layer. This correspond to our run $\mathrm{C}$ at $512^{2} \times 256$. The different mechanisms producing turbulence in convective and conditional neutral boundary layers (Moeng and Sullivan 1994), and the difference in the resulting characteristic turbulence scales, is thus of significant importance when studying mesh influence. Without going into details of all the budget terms in the turbulent kinetic energy equation, we show in Fig. 6 the ratio between resolved shear production, $\mathrm{SP}=-\left(\left\langle\tilde{u}^{\prime} \tilde{w}^{\prime}\right\rangle \partial\langle\tilde{u}\rangle / \partial z+\left\langle\tilde{v}^{\prime} \tilde{w}^{\prime}\right\rangle \partial\langle\tilde{\boldsymbol{v}}\rangle / \partial z\right)$ - the only term creating turbulent kinetic energy-and the dissipation, $\langle\mathscr{E}\rangle$, derived from the Deardorff (1980) SGS model: 

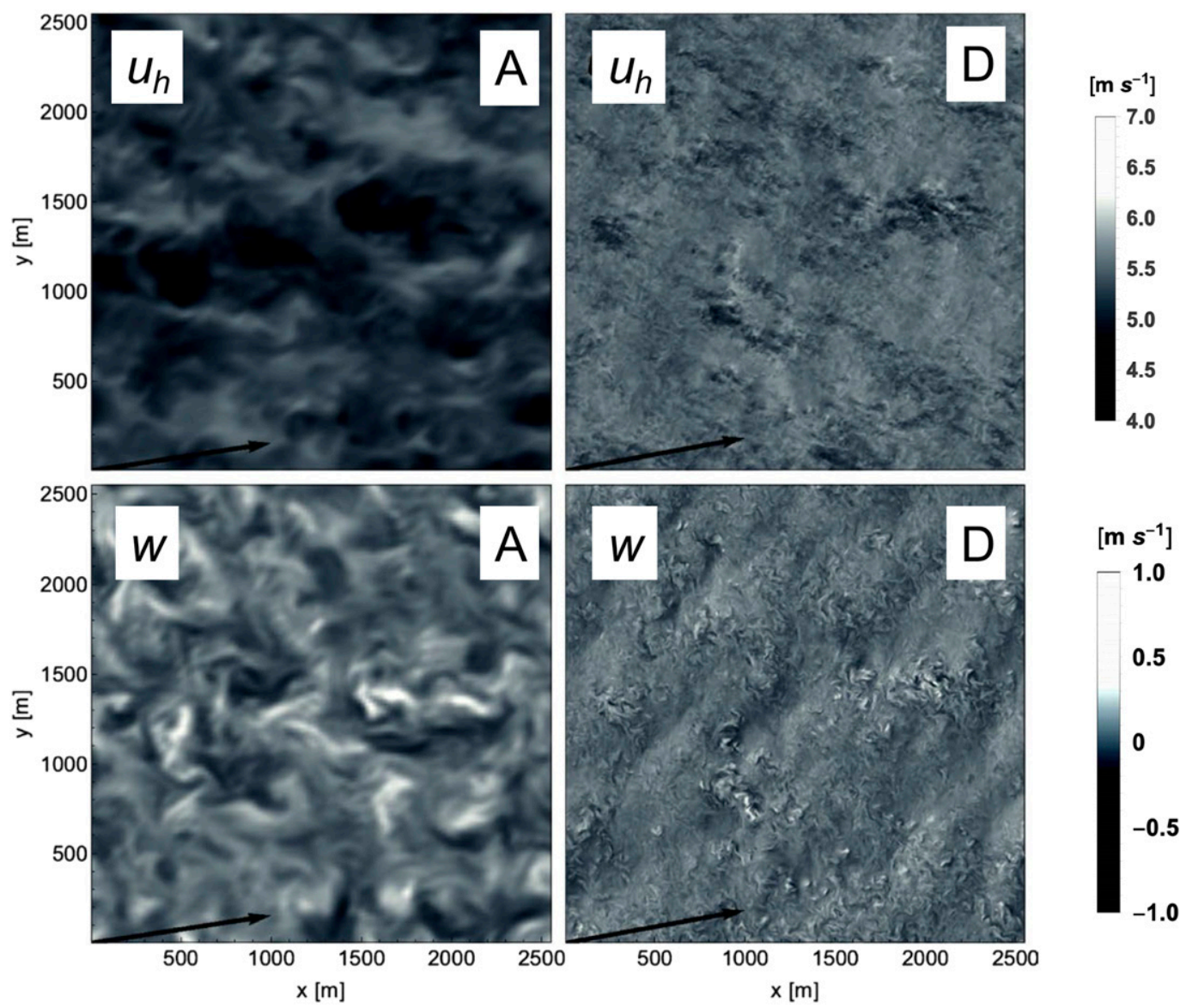

FIG. 8. As in Fig. 7, but for $z / z_{i}=0.9$. The color bars have been changed compared Fig. 7 .

$$
\langle\mathscr{E}\rangle=\left\langle\left(0.19+0.74 \frac{l}{\Delta_{f}}\right) \frac{e^{3 / 2}}{l}\right\rangle,
$$

where $l$ is a stability-limited length scale calculated according to $l=\min \left[l^{\star}, \Delta_{f}\right]$ with $l^{\star}=0.67 \sqrt{e / N^{2}}$. In principle there is also production of turbulent kinetic energy through a subfilter shear production $\left\langle\tau_{i j} \partial \tilde{u}_{i}^{\prime} / \partial x_{j}\right\rangle$ but since this term is balanced by an equal amount of turbulent kinetic energy transferred from the resolved scales to the subfilter scales, we do not consider it here. The ratio $\mathrm{SP} /\langle\mathscr{E}\rangle$ shows heights at which a local balance between shear production and dissipation is achieved. This height increases with decreasing resolution. For the coarsest simulation $\mathrm{A}$ it is never achieved, and even for the finest resolution runs, $\mathrm{C}$ and $\mathrm{D}$, is the height interval very narrow. For runs $\mathrm{C}$ and $\mathrm{D}$ it is found at $z / z_{i} \sim 0.1$. We will return to this balance when looking at structure functions later in the paper.

Very small dissipation rates are found near the ABL top $\left(z / z_{i}=0.9\right)$ for all four simulations. Strictly speaking, run $\mathrm{D}$ is the only simulation where $\left\langle l^{\star}\right\rangle / \Delta_{f}>1$ at $z / z_{i}=0.9$ (not shown), hence only in run $\mathrm{D}$ is there sufficient resolution to resolve the smallest scales for the problem design. At the larger-scale end of the eddy spectrum, the DoughertyOzmidov length scale, $L_{o}=\sqrt{\langle\mathscr{E}\rangle /\left\langle N^{2}\right\rangle^{3 / 2}}$ (Dougherty 1961; Ozmidov 1965), provides a measure of eddy sizes $\ell$ for which overturning would be inhibited $\left(\ell>L_{o}\right)$ by the strong stratification arising from entrainment of warm air from the "free" atmosphere above and turbulent mixing throughout the ABL. At $z / z_{i}=0.9$ we find $L_{o} / \Delta_{f}=\{0.2$, $0.5,1.2,2.4\}$, which means that only in runs $C$ and $D$ are the smallest scales unaffected by the strong stratification. Due to very high values of $L_{o}$ as we approach the surface where $N^{2}$ vanishes, for all simulations at $z / z_{i}<0.75$, we find that $\ell<L_{o}$ for all resolved eddies.

Instantaneous horizontal $(x-y)$ slices of streamwise velocity $u_{h}$ and vertical velocity $w$ reveal significant differences in the qualitative patterns between the coarsest and finest simulations (Figs. 7, 8). Here, streamwise velocity is defined $u_{h}=(\tilde{u}, \tilde{v}, 0) \cdot \hat{\mathbf{k}}_{h}$, where the streamwise vector (in the direction of the mean wind) is defined as $\hat{\mathbf{k}}_{h}=(\langle\tilde{u}\rangle,\langle\tilde{\boldsymbol{v}}\rangle) /|(\langle\tilde{u}\rangle,\langle\tilde{\boldsymbol{v}}\rangle)|$. Figure 7 presents snapshots at $z / z_{i}=0.1$, while height $z / z_{i}=0.9$, is presented in Fig. 8. 

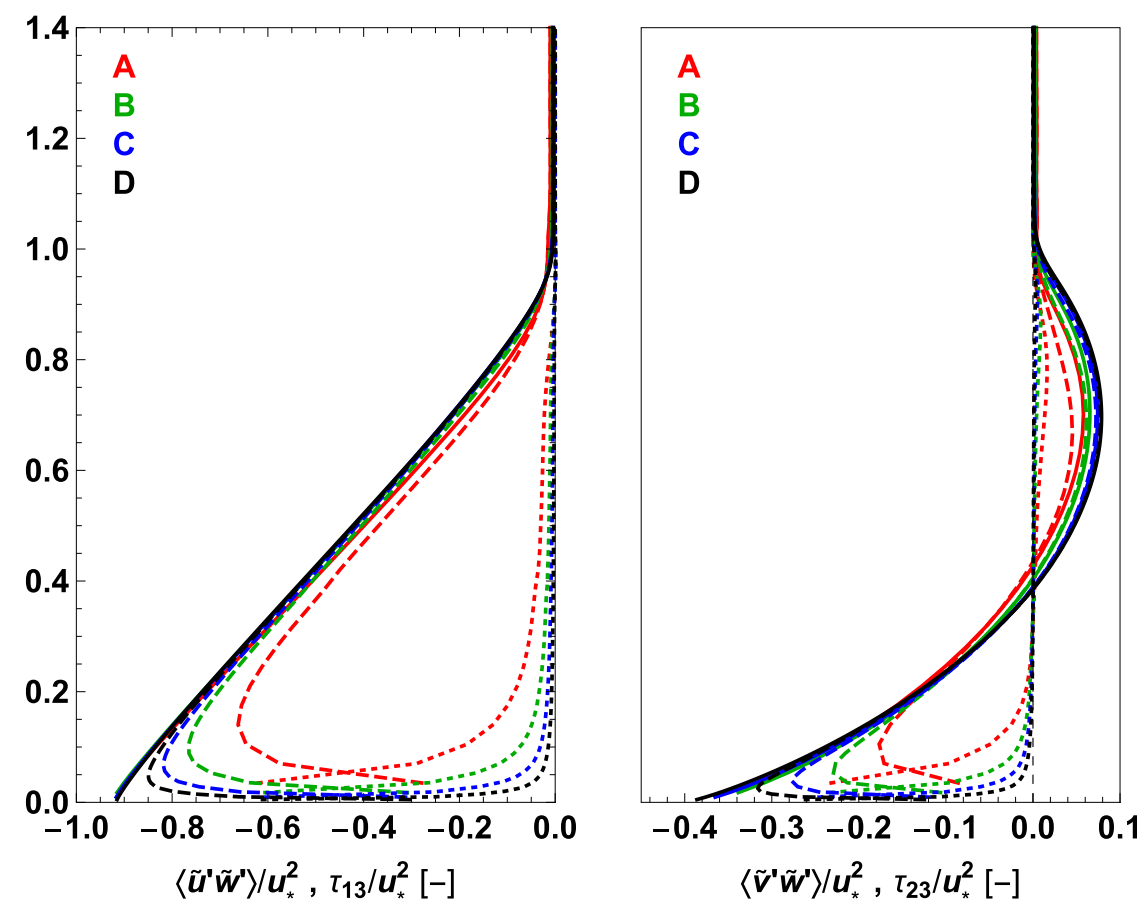

FIG. 9. Vertical momentum flux profiles: resolved, $\left\langle\tilde{u}_{i}^{\prime} \tilde{w}^{\prime}\right\rangle$ (dashed); SGS, $\left\langle\tau_{i 3}\right\rangle$ (dotted); and total (solid) for (left) $i=1$ and (right) $i=2$ normalized with $u_{\star}^{2}$.

A comparison of the horizontal and vertical velocity fields from simulations $\mathrm{A}$ and $\mathrm{D}$, collected at the same nondimensional vertical location, shows a dramatic increase in the intensity and number of small-scale eddies with increasing resolution. This is expected given the increase in large eddy Reynolds number in run D (e.g., Jiménez 2012). The abundant small-scale eddies in run D blur the relatively smooth large-scale velocity patterns readily observed in run A. The impact of increased resolution on vertical velocity is noticeably pronounced.
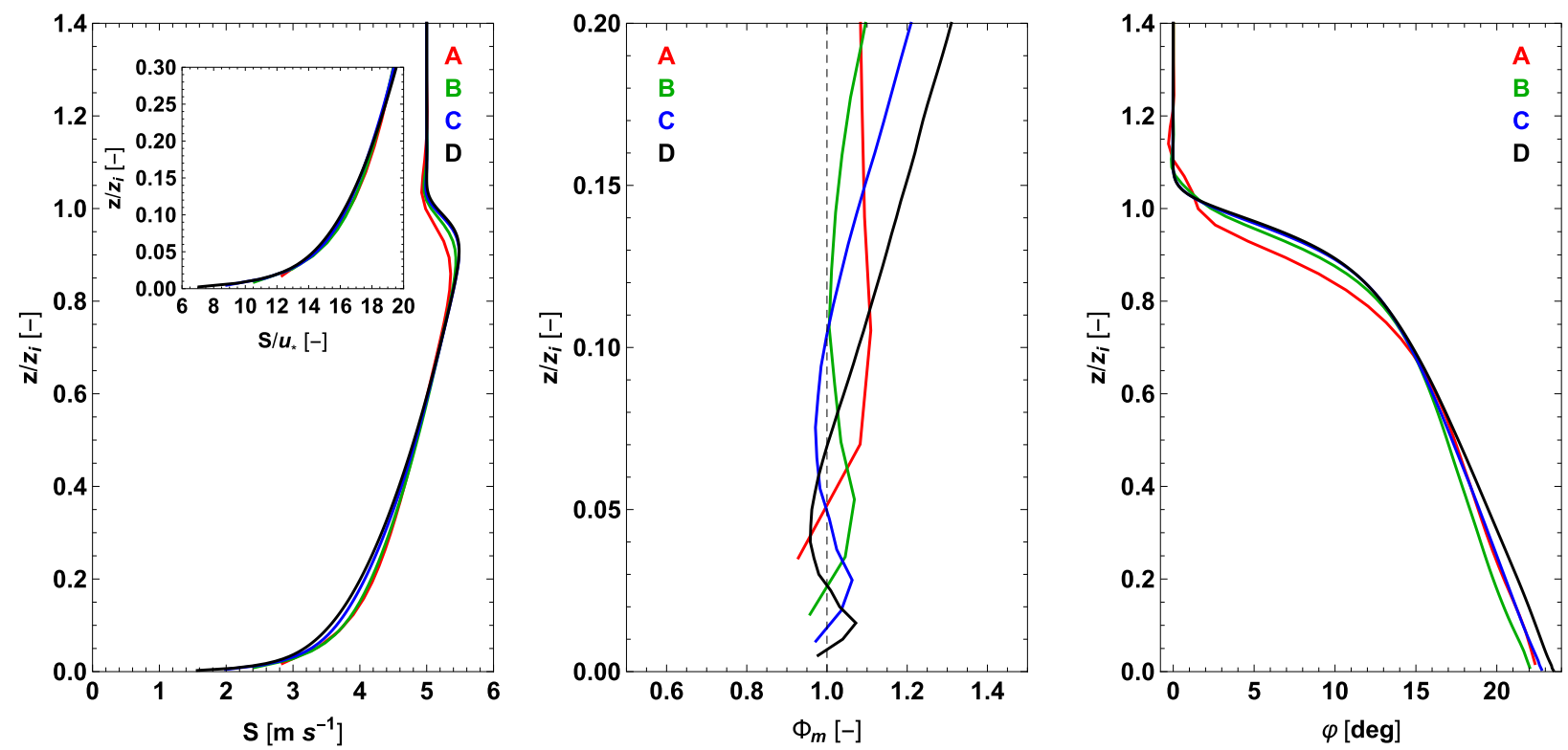

FIG. 10. Vertical profiles of (left) mean horizontal wind speed $S$ (inset is $S / u_{\star}$ vs $z / z_{i}$ ), (center) nondimensional wind shear $\phi_{m}$, and (right) wind direction $\varphi$ as a function of $z / z_{i}$. 

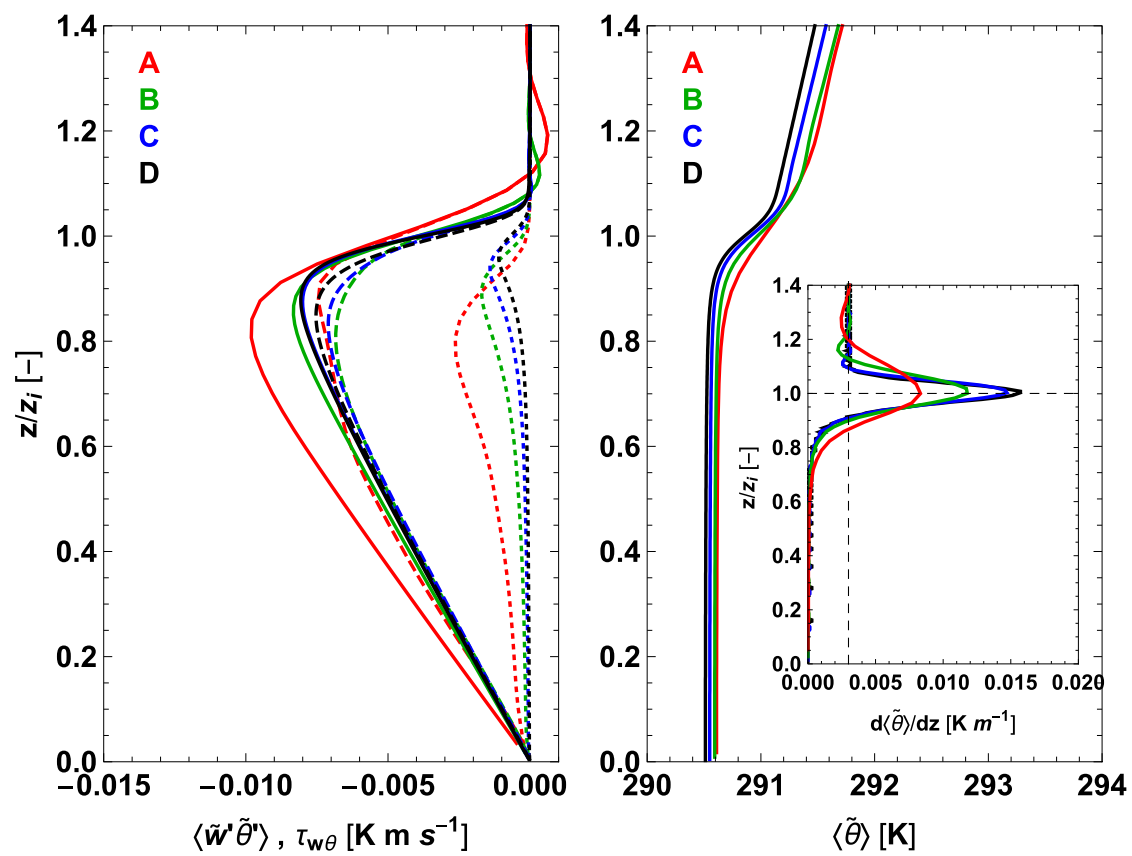

FIG. 11. Vertical profiles of horizontally and time-averaged (left) vertical heat flux and (right) temperature $\langle\tilde{\theta}\rangle$ as a function of $z / z_{i}$ and $z$, respectively. the heat flux is shown as follows: resolved, $\left\langle\tilde{w}^{\prime} \tilde{\theta}^{\prime}\right\rangle$ (dashed); SGS, $\left\langle\tau_{\theta 3}\right\rangle$ (dotted); and total (solid).

At $z / z_{i}=0.9$, the inability of run A to resolve the small scales associated with the buoyant destruction of turbulent kinetic energy as previously discussed is evident: the near intermittent velocity patterns observed in run $\mathrm{D}$ are qualitatively different from run A. A slight change in the main wind direction is also observed between the two resolutions at all heights and is due to the inability to resolve the turbulent momentum fluxes and shifts in $z_{i}$,
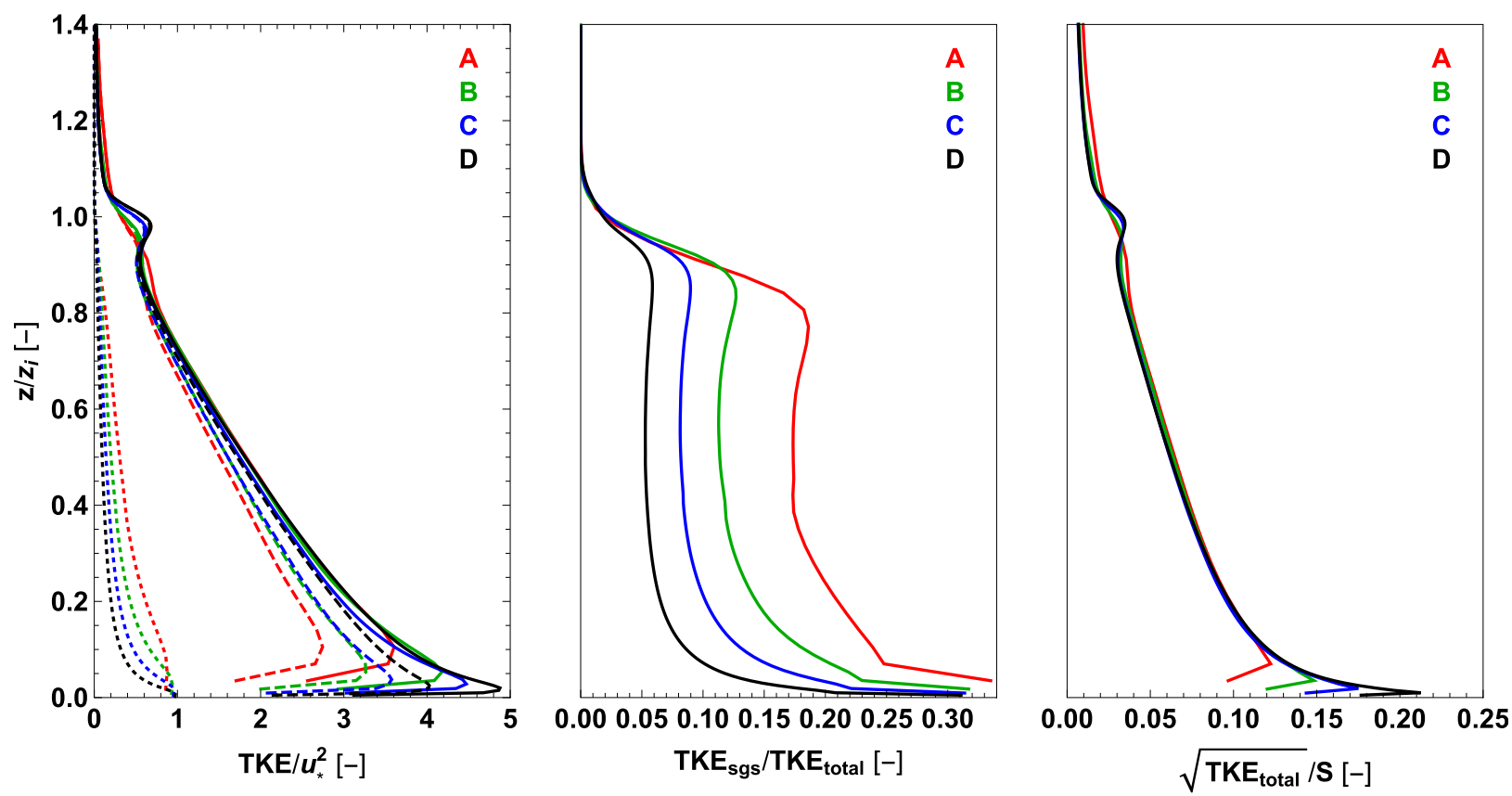

FIG. 12. Vertical profiles of (left) nondimensional TKE, (center) ratio of SGS to the total TKE, and (right) $\sqrt{\mathrm{TKE}} / S$ as a function of $z / z_{i}$. Lines are as follows: resolved (dashed), SGS (dotted), and total (solid). 

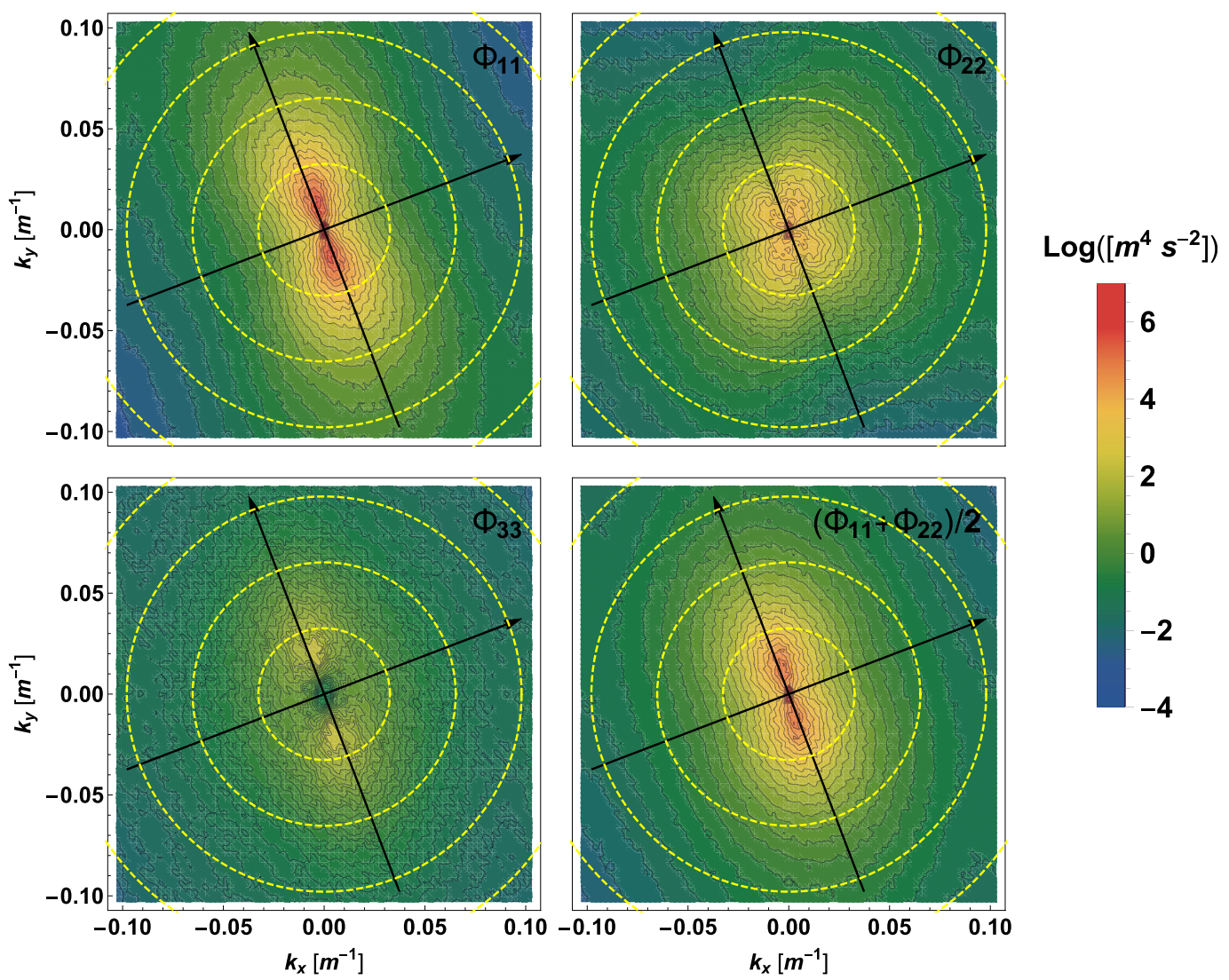

FIG. 13. Contour plots of the spectral tensor diagonal terms $\Phi_{i i}$ at $z / z_{i}=0.1$ for the $128^{2} \times 64$ (run A) simulation: (top left) $\Phi_{11}$, (top right) $\Phi_{22}$, (bottom left) $\Phi_{33}$, and (bottom right) $\left(\Phi_{11}+\Phi_{22}\right) / 2$. The contour lines are logarithmically scaled. The black lines are aligned with the streamwise and spanwise wavenumbers $k_{1}$ and $k_{2}$. The yellow rings are lines of constant $k_{h}$.

which both influence the wind veer with height (see section $4 b)$.

\section{b. Profiles of first- and second-order moments}

As previously mentioned, the turbulent momentum fluxes play a significant role in producing turbulence (viz. the importance of shear production in the turbulence kinetic energy budget). Figure 9 shows the resolved $\left\langle\tilde{u}_{i}^{\prime} \tilde{w}^{\prime}\right\rangle$, SGS $\left\langle\tau_{i 3}\right\rangle$, and the total momentum fluxes. Except for simulation A, profiles of the total momentum flux in the two horizontal directions (solid lines, Fig. 9) do not change substantially with resolution changes for heights $z / z_{i}>0.2$. On the other hand, the ratio between the resolved and SGS contributions increases significantly as the resolution increases. The first grid point at which the momentum fluxes are evaluated is at $z=\Delta z$. Whereas the magnitude of the total momentum flux vector at this level only differ by $2 \%$ between run A and run $\mathrm{D}$, the angle $\tan ^{-1}\left(\left\langle\tilde{v}^{\prime} \tilde{w}^{\prime}+\tau_{23}\right\rangle /\left\langle\tilde{u}^{\prime} \tilde{w}^{\prime}+\tau_{13}\right\rangle\right)$ is more different. One finds a $3^{\circ}$ difference between runs $\mathrm{A}$ and $\mathrm{D}$ (the angle in run $\mathrm{D}$ is $22.9^{\circ}$ ). If we on the other hand only look at the resolved contribution, $\tan ^{-1}\left(\left\langle\tilde{\boldsymbol{v}}^{\prime} \tilde{w}^{\prime}\right\rangle /\left\langle\tilde{u}^{\prime} \tilde{w}^{\prime}\right\rangle\right)$, we find a $5^{\circ}$ difference. That is, the run $\mathrm{D}$ is capable of resolving relatively more of the smaller-scale $\left\langle\tilde{v}^{\prime} \tilde{w}^{\prime}\right\rangle$ covariance compared to the coarser run A. Since the momentum flux vector is aligned with the vector of the mean shear, $(d\langle\tilde{u}\rangle / d z, d\langle\tilde{v}\rangle / d z)$, this has implications for the wind direction (Berg et al. 2013). A change in mean wind direction was observed in Figs. 7 and 8 when comparing run $\mathrm{A}$ and run $\mathrm{D}$.

The mean wind $S$ is shown in Fig. 10 (left panel). All simulations produce a supergeostrophic velocity with maximum speed location increasing with increasing resolution $\left(z / z_{i} \sim 0.85\right.$ for run $\mathrm{A}$ to $z / z_{i} \sim 0.93$ for run $\left.\mathrm{D}\right)$. Some discrepancy between simulations can be seen in the near-surface mean wind profiles, but they become more similar after rescaling the profiles with each simulation's respective $u_{\star}$, at least up to around $z / z_{i} \sim 0.2$. Visualization of true logarithmic scaling (which should only exist in true shear-driven boundary layers, i.e., in the absence of stratification and Coriolis forces), is best performed by looking at the nondimensional wind shear, 

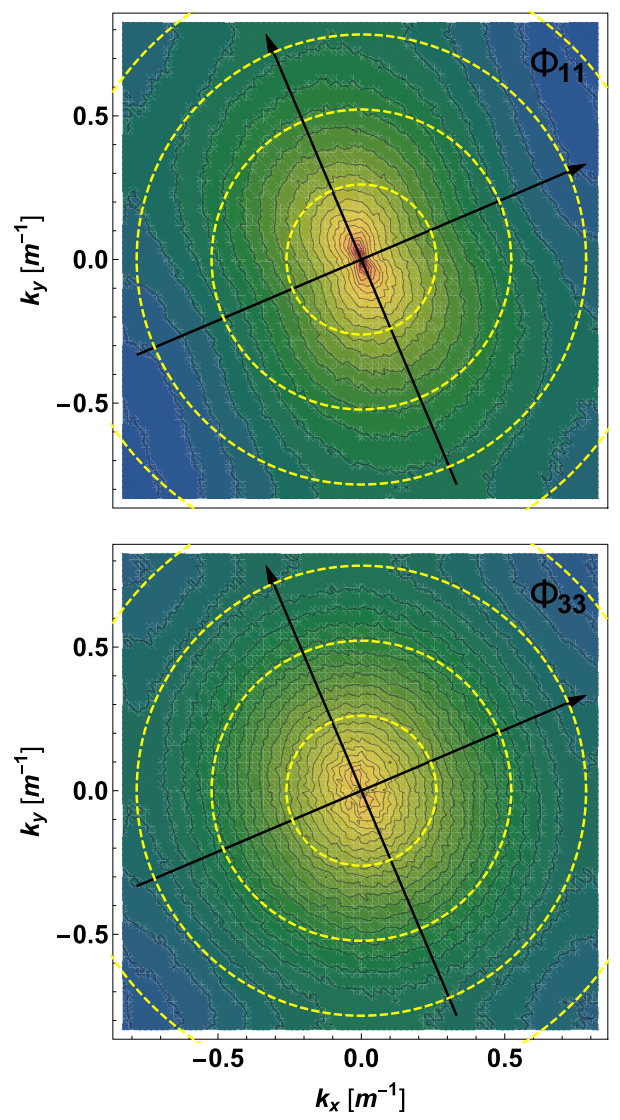
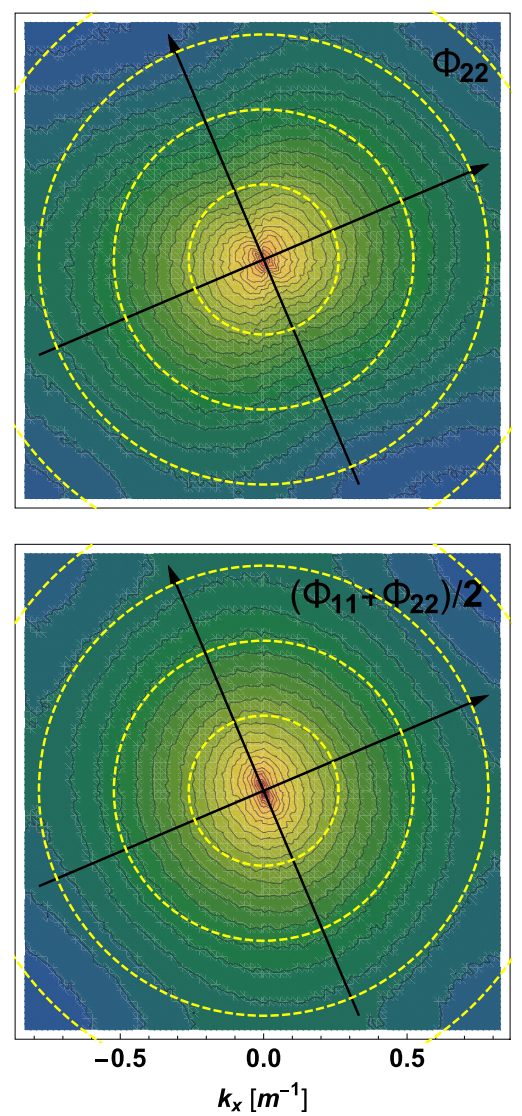

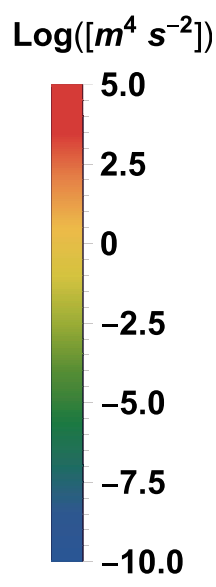

FIG. 14. As in Fig. 13, but for the $1024^{2} \times 512$ (run D) simulation. $\phi_{m}=\left(\kappa z / u_{\star}\right)(\partial S / \partial z)$ in close proximity to the lower boundary; $\phi_{m}$ is plotted in Fig. 10 (center panel), where a true near-surface logarithmic layer should appear as a region where $\phi_{m}=1$. Near-surface departure from $\phi_{m}=1$ (i.e., the overshoot) is constant in magnitude for all runs but shifts toward the surface with increasing resolution, a result consistent with Brasseur and Wei (2010). The relatively low magnitude of the overshoot (less than $10 \%$ ) is attributed to application of Sullivan et al.'s (1994) two-part SGS model. Especially for the two highest-resolution simulations (runs $\mathrm{C}$ and D), the influence of the stable stratification appears as a constant increase in $\phi_{m}$ from $z / z_{i} \sim 0.08$ and up.

In Fig. 10 (right panel) we show the mean wind direction $\varphi$ for the four runs. Stronger veering (wind direction change with height) with increased resolution is observed. This is in agreement with the findings just reported on the angle between resolved momentum fluxes.

We now turn to the heat flux profiles in Fig. 11 (left panel). For all resolutions a close to linear profile from the surface to the minimum value of heat flux is observed. The minimum value decreases and the height of this minimum increases with increasing resolution. This is in agreement with the DNS study by Jonker et al. (2013). In their DNS no "overshoot" is observed above $z_{i}$. Such "overshoot" is present in our coarse-resolution runs $\mathrm{A}$ and $\mathrm{B}$ but diminishes in the fine-resolution runs $\mathrm{C}$ and D. Looking at the SGS contribution $\left\langle\tau_{w \theta}\right\rangle$, we see that the minimum value for simulation $\mathrm{A}$ ocurrs at a height below the minimum of the total heat flux compared to runs $\mathrm{C}$ and $\mathrm{D}$, where it is located above the minimum of the total flux. Since the heat flux is ultimately linked to the gradient of potential temperature, we show the profile of $\langle\tilde{\theta}\rangle$ in Fig. 11 (right panel). In the inset we observe how the coarse resolution fails to resolve the gradient and how it instead "smears" it out over a larger depth compared to the fine resolution runs. Due to the increased heat flux in the coarse simulations the bulk ABL temperature increases slightly with decreasing resolution $(0.1 \mathrm{~K})$.

Figure 12 presents the total turbulent kinetic energy, TKE $=\left\langle\tilde{u}_{i}^{\prime} \tilde{u}_{i}^{\prime}\right\rangle / 2+\langle e\rangle$, where summation is implied and $e$ is the SGS turbulent kinetic energy in Eq. (4). In the left panel the total TKE converges across simulations throughout much of the ABL except at $z / z_{i} \sim 1$, where turbulence is produced by shear associated with the 

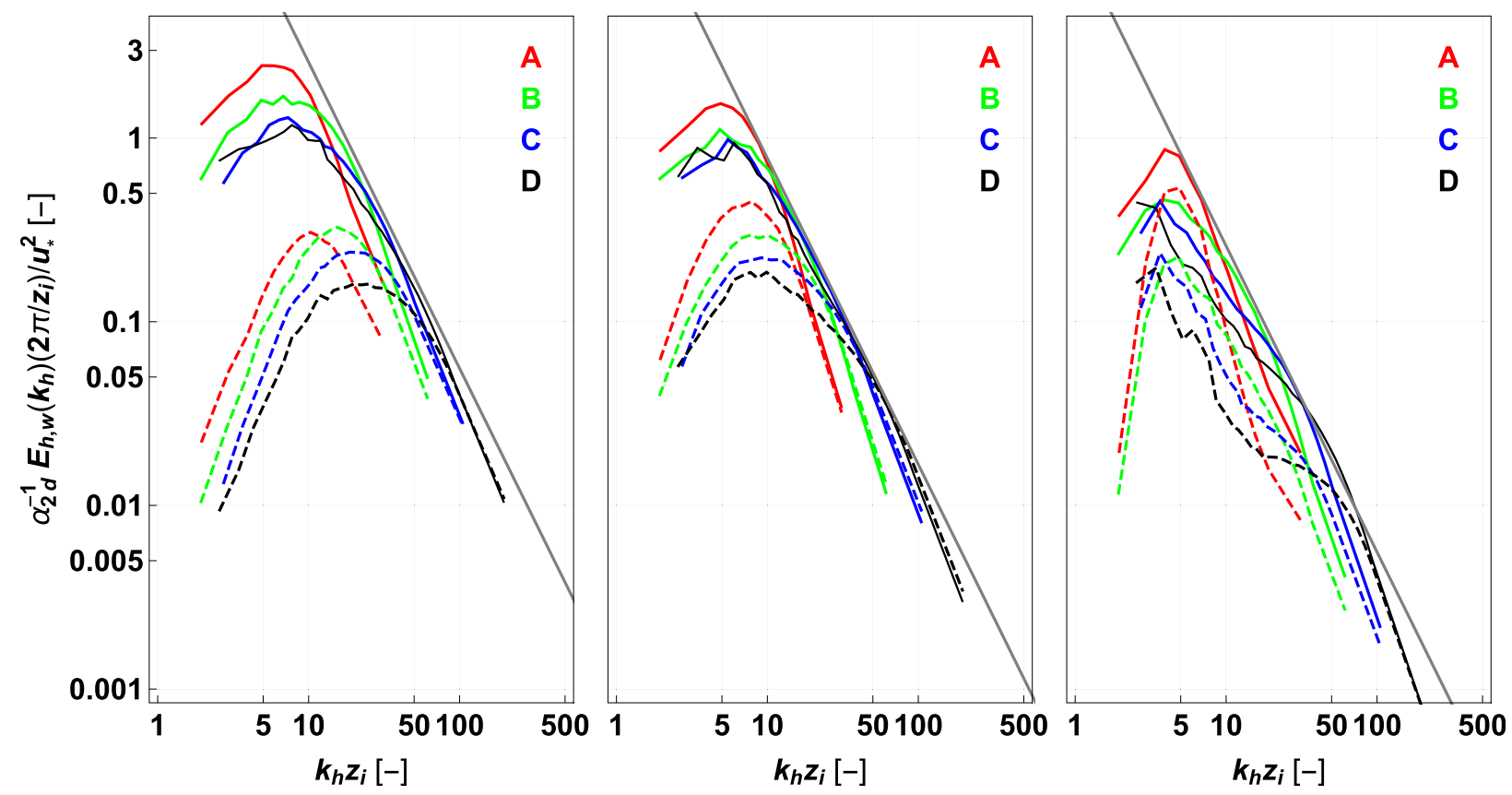

FIG. 15. Two-dimensional horizontal spectra $E_{h}\left(k_{h}\right)$ (solid) and vertical spectra $E_{w}\left(k_{h}\right)$ (dashed) for $z / z_{i}=($ left) 0.1 , (center) 0.5 , and (right) 0.9. All spectra have been nondimensionalized with a factor $2 \pi z_{i}^{-1} u_{\star}^{-2}$.

supergeostrophic jet and destroyed by buoyancy, which are both highly dependent on the mesh resolution due to the small scales involved: we observe that the smaller jet observed in simulation A (Fig. 10 do not give rise to the same amount of turbulence as for the other more resolved runs. Close to the surface, increasing mesh resolution naturally resolves the increasingly smaller scales. Convergence throughout the ABL of total TKE implies commensurate altering of the partitioning between resolved and SGS energy with mesh resolution variations. The contribution from SGS energy is increasing with approximately $50 \%$ each time the filter $\Delta_{f}$ is doubled.

The right panel of Fig. 12 shows a surrogate for turbulence intensity (i.e., $\sqrt{\mathrm{TKE}} / S$ ). We choose this definition instead of the wind-energy community's usual definition: $\sigma_{u_{h}} /\left|u_{h}\right|$, where $\sigma_{u_{h}}$ denotes the standard deviation of the streamwise velocity component; however, since $\sigma_{v} \sim 0.8 \sigma_{u}$ and $\sigma_{w} \sim 0.5 \sigma_{u}$, (Panofsky and Dutton 1984), the difference between the two definitions should only result in magnitude differences of $\sim 3 \%$. Again we observe mesh independence throughout the ABL except at $z / z_{i} \sim 1$ and close to the surface. In wind energy meteorology such a result could potentially be quite useful: even coarse-resolution LES can produce sufficiently accurate turbulence intensity predictions to determine appropriate wind turbine classes for siting purposes (e.g., International Electrotechnical Commission 2005), where for this configuration coarse resolution means $\Delta_{f}=23.3 \mathrm{~m}$. Close to the surface the SGS model fails to deliver the part of TKE missing due to unresolved eddies, and hence the somewhat bold statement above also do not apply to turbines operating in the surface layer.

\section{c. The spectral tensor and inertial range scaling}

Further insight into the second-order statistics can be gained by looking at the spectral properties of the simulations. As a starting point, the spectral tensor in stationary and horizontal homogeneous conditions is

$$
\Phi_{i j}\left(k_{x}, k_{y}, z\right)=\frac{1}{(2 \pi)^{2}} \iint R_{i j}\left(r_{x}, r_{y}, z\right) e^{-l\left(k_{x} r_{x}+k_{y} r_{y}\right)} d r_{x} d r_{y}
$$

with the covariance function,

$$
R_{i j}\left(r_{x}, r_{y}, z\right)=\left\langle\tilde{u}_{i}^{\prime}(x, y, z) \tilde{u}_{j}^{\prime}\left(x+r_{x}, y+r_{y}, z\right)\right\rangle,
$$

and horizontal wavenumbers, $\mathbf{k}=\left(k_{x}, k_{y}\right)$, aligned with the LES coordinate system. The spectral tensor has the properties, $\Phi_{i j}(\mathbf{k}, z)=\Phi_{i i}^{\star}(\mathbf{k}, z)$ and $k_{i} \Phi_{i j}(\mathbf{k}, z)=0$, where the pentagram denotes complex conjugation. To account for the fact that the wind direction is not aligned with the coordinate axis, the spectral tensor has been rotated at each height, $\Phi_{i j}^{\mathrm{rot}}=\mathbf{M}_{i k} \Phi_{k l} \mathbf{M}_{j l}$, where $\mathbf{M}_{i j}$ is the matrix that rotates a wind vector into the mean horizontal wind direction, so that $k_{1}$ is in the direction of the mean wind at each height. 

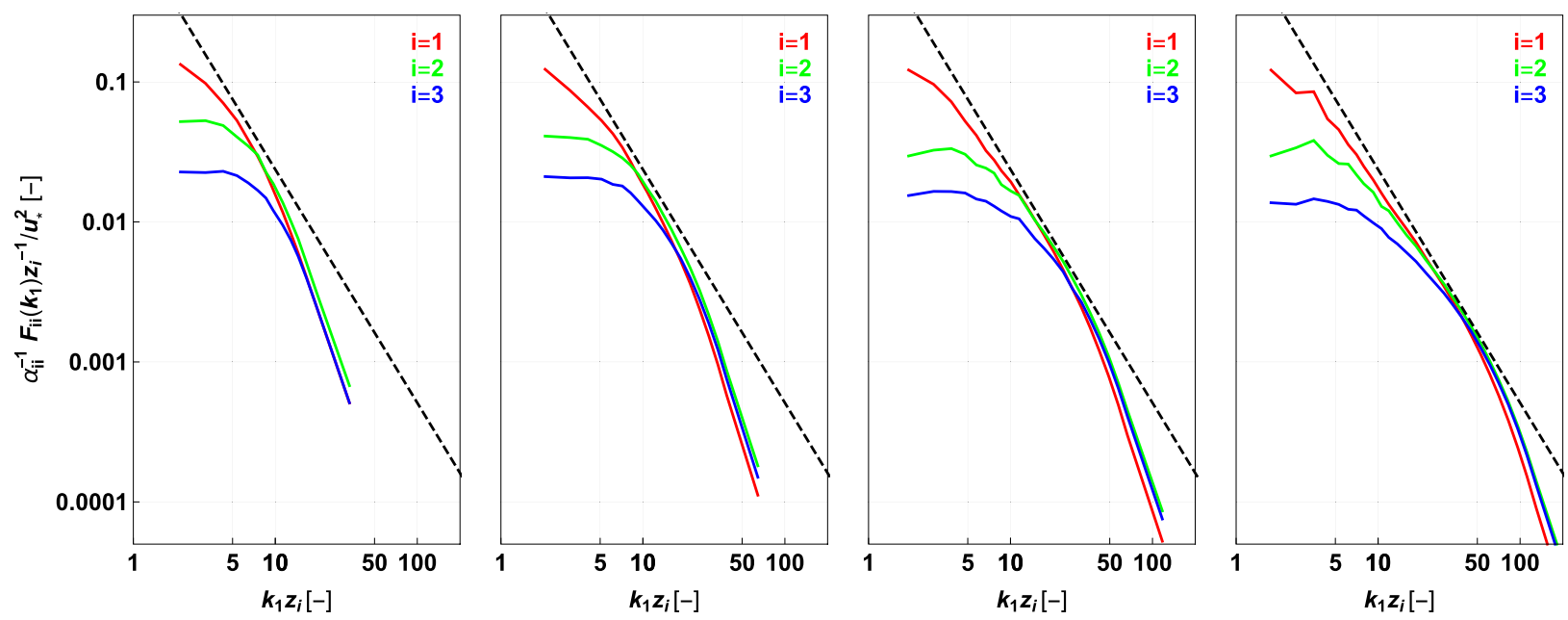

FIG. 16. One-dimensional spectra $F_{i i}\left(k_{1}\right)$ at $z / z_{i}=0.5$ for $i=1$ (red lines), $i=2$ (green lines), and $i=3$ (blue lines) for (left) run A, (left center) run $\mathrm{B}$, (right center) run $\mathrm{C}$, and (right) run $\mathrm{D}$. All spectra have been nondimensionalized with a factor $z_{i}^{-1} u_{\star}^{-2}$.

Figures 13 and 14 show the diagonal terms of the spectral tensor for the $128^{2} \times 64$ (run A) and $1024^{2} \times$ 512 (run D) simulations at $z / z_{i}=0.1$, respectively. The main features in the spectral tensors clearly align with the streamwise and spanwise wavenumbers $k_{1}$ and $k_{2}$ for the two horizontal terms $\Phi_{11}$ and $\Phi_{22}$, respectively; this result is especially true for the high-resolution simulation $\mathrm{D}$, whereas some misalignment is observed for the coarser simulation A. The mean wind is associated with lowest wavenumber (i.e., in the center of the plots); the misalignment increases with increasing wavenumber such that the smallest scales exhibit the
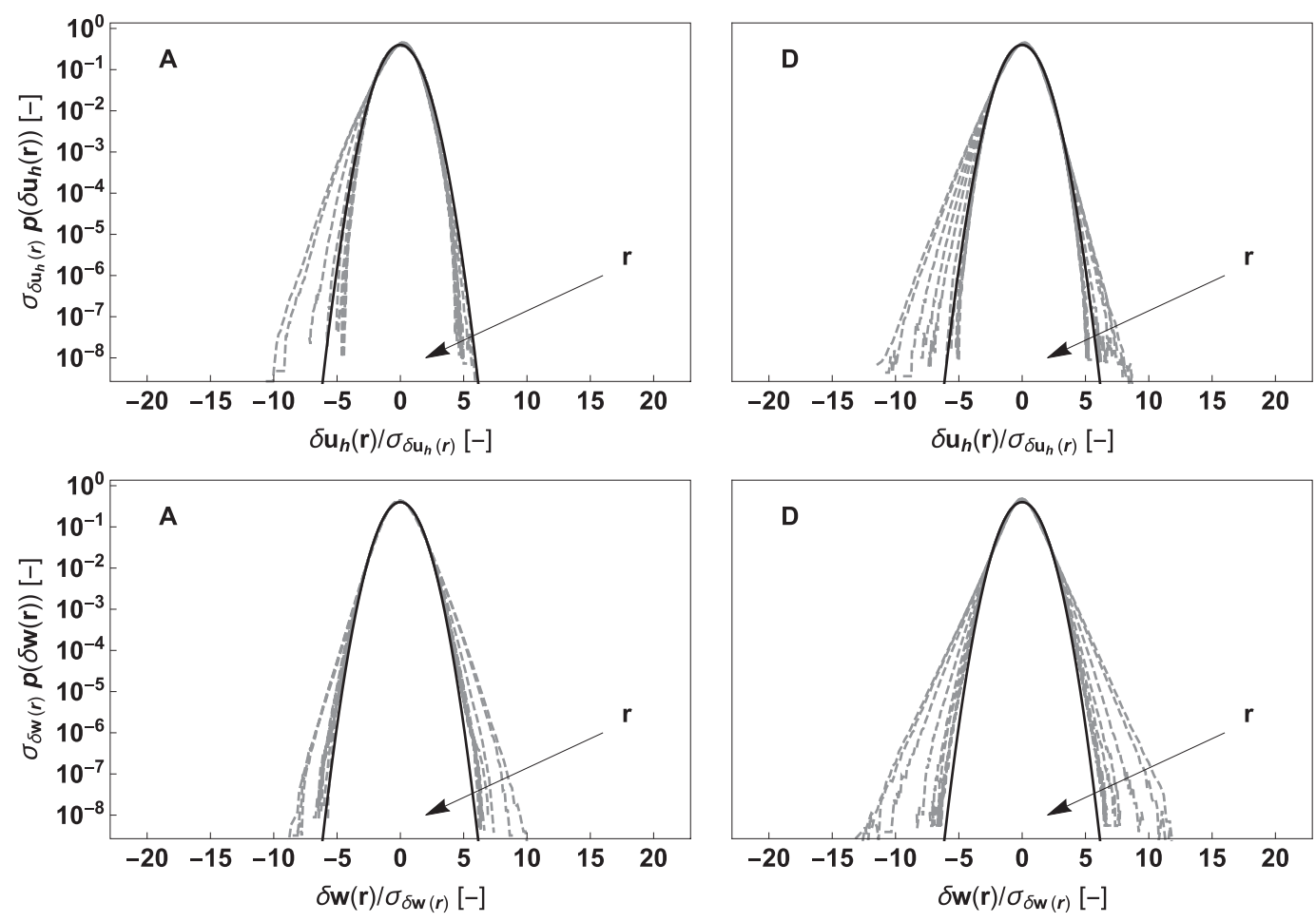

FIG. 17. Pdfs of velocity increments in the streamwise direction $p(\cdot)$ at $z / z_{i}=0.1$ for (top) the streamwise component and (bottom) the vertical component in (left) the coarse simulation (run A) and (right) the fine simulation (run D). The thick black line, representing $-x^{2}$, is the Gaussian distribution. We plot pdfs for separations, $r \in\left[\Delta x, \ldots, L_{x} / 2\right]$. 

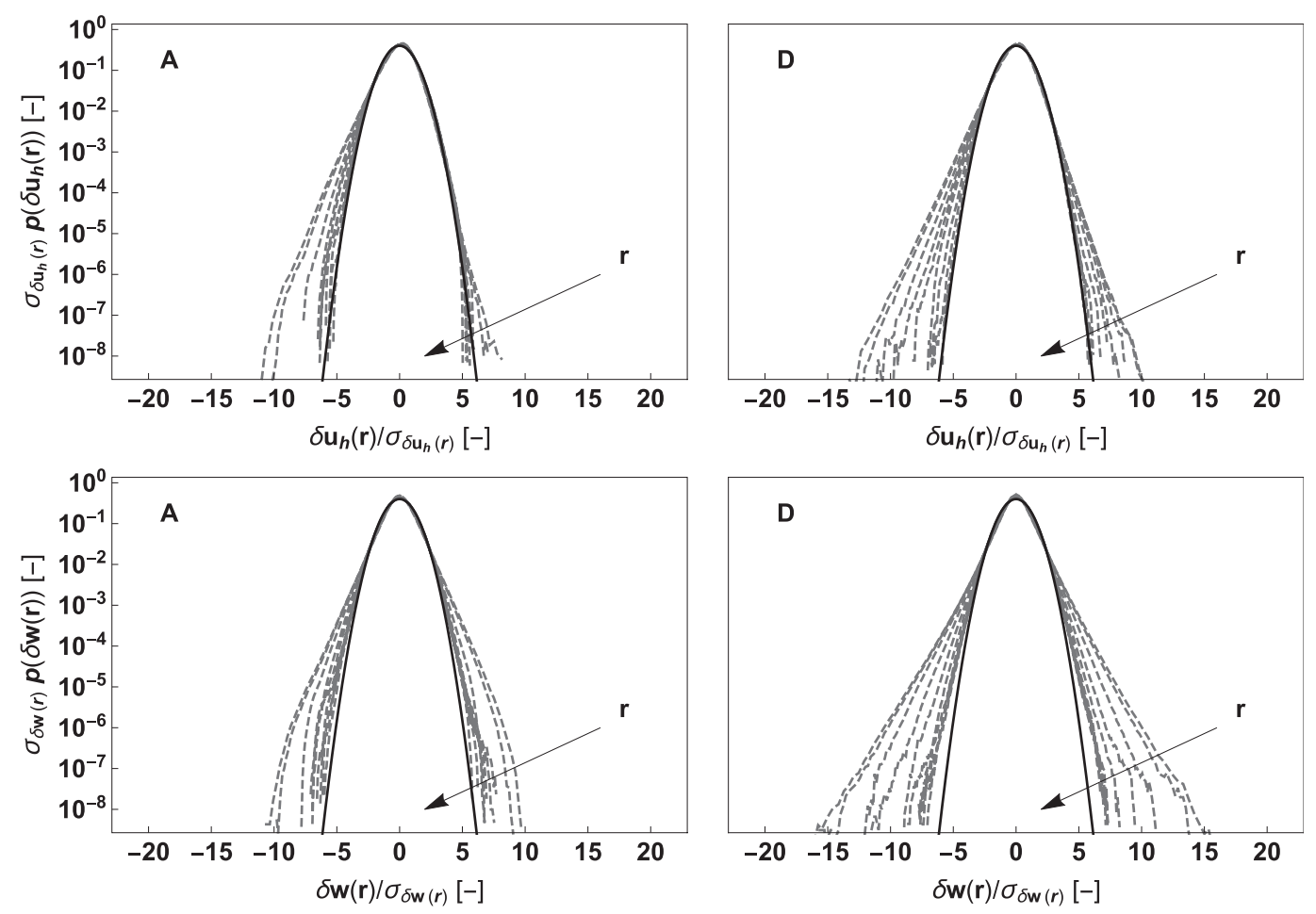

FIG. 18. As in Fig. 17, but for $z / z_{i}=0.5$.

largest misalignment. Again, this result is most pronounced in run $\mathrm{A}$.

The average of $\Phi_{11}$ and $\Phi_{22}$ shows the azimuthal symmetry [and hence follows the incompressibility constraint, $k_{i} \Phi_{i j}(\mathbf{k})=0$ in Pope $(2000$, p. 220)], for all but the smallest scales in run D, which makes $\left(\Phi_{11}+\right.$ $\left.\Phi_{11}\right) / 2$ an obvious candidate for azimuthal averaging (also denoted as "ring averaging"). Azimuthal symmetry is also observed in the vertical component $\Phi_{33}$ Run A does not display azimuthal symmetry for either $\left(\Phi_{11}+\Phi_{22}\right) / 2$ or $\Phi_{33}$.

Following Peltier et al. (1996), Kelly and Wyngaard (2006), and Wyngaard (2010) we exploit the symmetry observed in $\Phi_{33}$ and $\left(\Phi_{11}+\Phi_{22}\right) / 2$ by calculating twodimensional ring-averaged horizontal velocity spectra $E_{h}\left(k_{h}\right)$ and vertical velocity spectra $E_{h}\left(k_{h}\right)$ as a function of horizontal wavenumber, $k_{h}=\left(k_{1}^{2}+k_{2}^{2}\right)^{1 / 2}$ :

$$
\begin{aligned}
& E_{h}\left(k_{h}\right)=\frac{1}{2} \int_{0}^{2 \pi}\left[\Phi_{11}\left(k_{h}, \varphi\right)+\Phi_{22}\left(k_{h}, \varphi\right)\right] k_{h} d \varphi, \\
& E_{w}\left(k_{h}\right)=\int_{0}^{2 \pi} \Phi_{33}\left(k_{h}, \varphi\right) k_{h} d \varphi .
\end{aligned}
$$

In the inertial subrange, the spectra are supposed to follow the dimensional " $-5 / 3$ " scaling, with $E_{h}\left(k_{h}\right)=$ $\alpha_{h} \mathscr{E}^{2 / 3} k_{h}^{-5 / 3}$ and $E_{w}\left(k_{h}\right)=\alpha_{w} \mathscr{E}^{2 / 3} k_{h}^{-5 / 3}$, with constants $\alpha_{h}=0.54(55 / 18) \alpha_{1 d}$, and $\alpha_{w}=0.61(55 / 18) \alpha_{1 d}($ Wyngaard
2010), and the one-dimensional Kolmogorov constant taken to be $\alpha_{1 d}=0.5$ (Sreenivasan 1995). Figure 15 presents ring-averaged two-dimensional spectra $E_{h}\left(k_{h}\right)$ and $E_{w}\left(k_{h}\right)$. At $z / z_{i}=0.1$ the horizontal spectra for runs C and $\mathrm{D}$ start to show an inertial subrange. Since the spectra have been multiplied by the inverse constants, $\alpha_{h}$ and $\alpha_{w}$, collapse between the horizontal and vertical spectra indicate that their mutual relationship is found to follow its theoretical prediction. This is the case at $z / z_{i}=0.1$ for runs $\mathrm{C}$ and $\mathrm{D}$. Moving to $z / z_{i}=0.5$ we see the extended range reasonably close to $-5 / 3$ scaling for all runs and a collapse of the two spectra. As $k z_{i}$ increases and the scales are being affected by the SGS model the slopes decline slightly. At $z / z_{i}=0.9$ all resolved scales in all the four runs (except the very smallest in run D affected by SGS dissipation) are larger than the Ozmidov scale $L_{o}$. Hence they are influenced by the stable stratification and therefore do not exhibit fully three-dimensional turbulence-together with the small scales present at this height inertial range scaling is inapplicable.

Compared to more typical one-dimensional spectra, that is,

$$
F_{i j}\left(k_{1}\right)=\int \Phi_{i j}\left(k_{1}, k_{2}\right) d k_{2},
$$

the advantage of interrogating two-dimensional ringaveraged spectra becomes apparent by studying the 

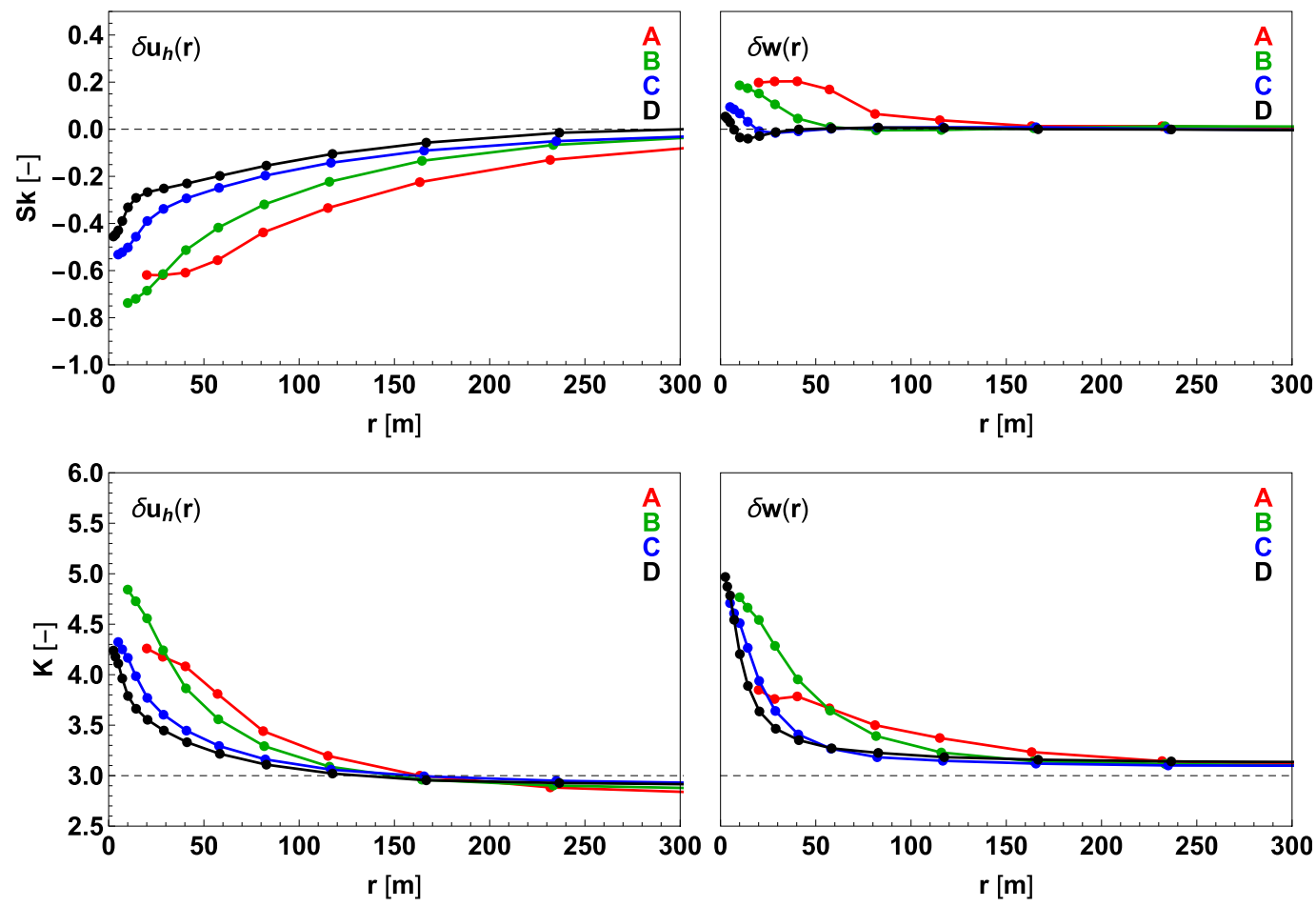

FIG. 19. Normalized (top) third moment Sk and (bottom) fourth moment, $K$ of the velocity increments as a function of horizontal separation distance $r$ and at a height of $z / z_{i}=0.1$ for (left) the streamwise velocity component and (right) the vertical velocity component.

spectral tensor plots (Figs. 13, 14). Averaging $F_{i j}\left(k_{1}\right)$ over $k_{2}$ introduces aliasing errors from spanwise motions. Furthermore, in situations including the Coriolis force, using a square domain with principle coordinates, $x$ and $y$, the integration limits change, since the structure of $\Phi_{i j}$ aligns along $k_{1}$ and $k_{2}$ as opposed to along $k_{x}$ and $k_{y}$, in effect smaller scales, are somewhat effected.

The disadvantage of using two-dimensional spectra lies in the lack of available measurements. Measurements tend to be performed in the temporal domain and after assuming Taylor's frozen-eddy hypothesis (Taylor 1938) and presented as one-dimensional spectra $F_{i j}\left(k_{1}\right)$ in the streamwise direction. Figure 16 shows one-dimensional spectra $F_{i i}\left(k_{1}\right)$, where the spectra have been multiplied with the inverse Kolmogorov constant $\alpha_{1 d}$ at $z / z_{i}=0.5$ (i.e., at the height that most clearly contained an inertial subrange in the $2 \mathrm{D}$ spectra according to Fig. 15). The three curves in each panel do not fully collapse in the inertial subrange, demonstrating that they are not fully consistent with (4/3) $F_{11}=F_{22}=F_{33}$ as one would expect for isotropic tensors (see Pope 2000, for example). The collapse of the curves improves with increasing resolution, but even at the finest resolution (run $\mathrm{D}$, right panel of Fig. 16), the range over which they collapse, and follow the $-5 / 3$ scaling law is restricted compared to its twodimensional counterpart. The $-5 / 3$ law is thus more difficult to discern in the one-dimensional spectra compared to two-dimensional spectra; a well known result that we attribute to sampling issues connected to $k_{x}-k_{y}$ versus $k_{1}-k_{2}$ as described above-an effect that is rarely highlighted in the literature. Recently is has also been documented by Ansorge (2019) how a systematic displacement of structures in the lowest part of the boundary layer dictates the use of two-dimensional filters only.

\section{d. Velocity increments, structure functions, and generalized log law}

Finally, structure function analysis allows interrogation of the Gaussianity (or non-Gaussianity) of velocity increment statistics in the streamwise and in the vertical directions. Streamwise velocity increments at a distance $r$ in the streamwise direction are defined as

$$
\delta u_{h}(r) \equiv \hat{\mathbf{k}}_{h} \cdot\left[\tilde{\mathbf{u}}^{\prime}\left(\mathbf{x}+r \hat{\mathbf{k}}_{h}, t\right)-\tilde{\mathbf{u}}^{\prime}(\mathbf{x}, t)\right],
$$

where $\hat{\mathbf{k}}_{h}$ is the streamwise unit vector defined previously, while

$$
\delta w(r) \equiv \tilde{w}^{\prime}\left(\mathbf{x}+r \hat{\mathbf{k}}_{h}, t\right)-\tilde{w}^{\prime}(\mathbf{x}, t)
$$

defines the vertical velocity increments. Normalized probability density functions (pdfs) of the velocity 

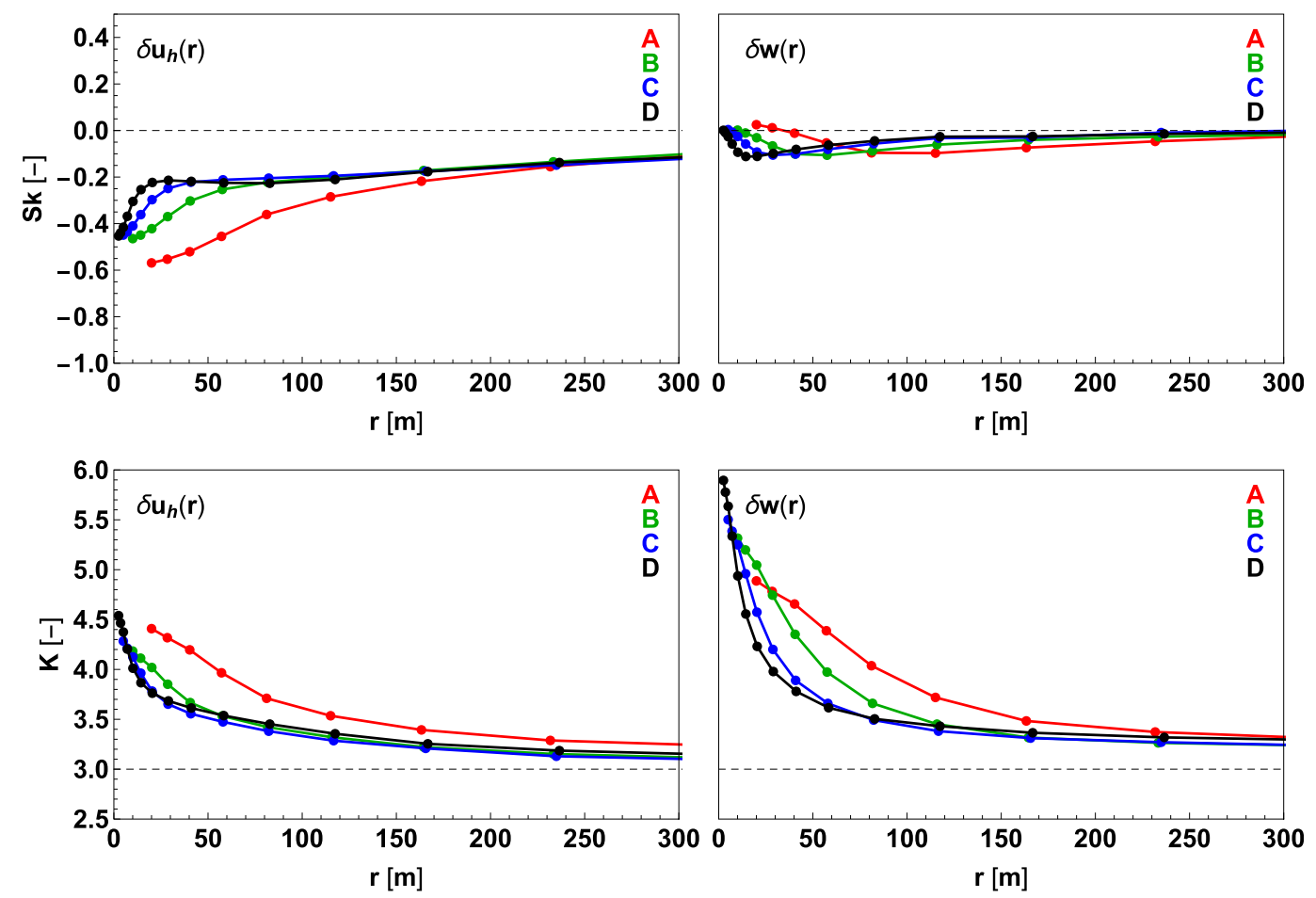

FIG. 20. As in Fig. 19, but for $z / z_{i}=0.5$.

increments (Figs. 17, 18 ) reveal some striking features. First of all the pdfs become more Gaussian with decreasing distance $r$ (i.e., departure from Gaussian statistics is most pronounced for small scales in the flow), which is in agreement with most previous observations and theory describing turbulent high-Reynolds-number flow (Frisch 1995). The pdfs of the finest-resolution simulation (run D) exhibit the broadest tails, while in the coarse simulation (run A) the pdf tails level-off in a concave shape indicating truncation caused by the SGS model. From the spectral analysis (section 4c), the inertial range for this mesh size $\left(128^{2} \times 64\right)$ is very narrow and hardly follows conventional scaling. Vertical velocity exhibits the largest non-Gaussianity, in agreement

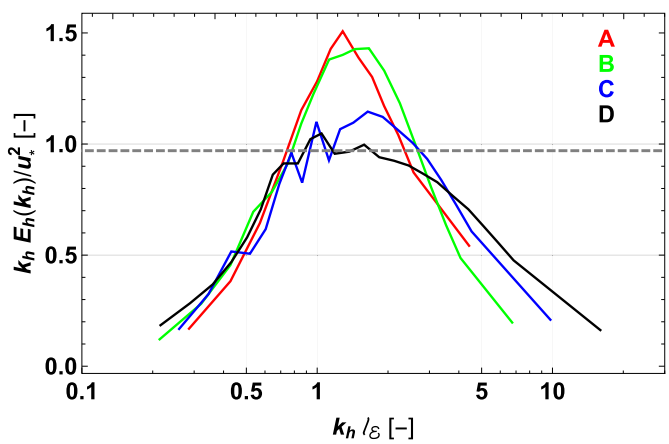

with visual inspection of the smallest scales in the instantaneous horizontal slices of vertical velocity in Figs. 7 and 8. Finally, the most non-Gaussian velocity increments in run A occurs at $z / z_{i} \sim 0.5$ compared to at $z / z_{i} \sim 0.1$ for run $\mathrm{D}$, which results from higher-resolution supporting a broader inertial range due to the large turbulent scales of motion present at $z / z_{i} \sim 0.5$.

Defining the structure function of $n$th order as ensemble averages $\left\langle\delta u_{h}^{n}(r)\right\rangle$ and $\left\langle\delta w^{n}(r)\right\rangle$ for the streamwise and vertical components, respectively, the third and fourth normalized moments (i.e., skewness $\mathrm{Sk}$ and kurtosis $K)$, are given by $\mathrm{Sk} \equiv\left\langle\delta u_{h}^{3}\right\rangle /\left\langle\delta u_{h}^{2}\right\rangle^{3 / 2}$ and $K \equiv\left\langle\delta u_{h}^{4}\right\rangle /\left\langle\delta u_{h}^{2}\right\rangle^{2}$ and similar for the vertical component. Velocity skewness and kurtosis are presented in

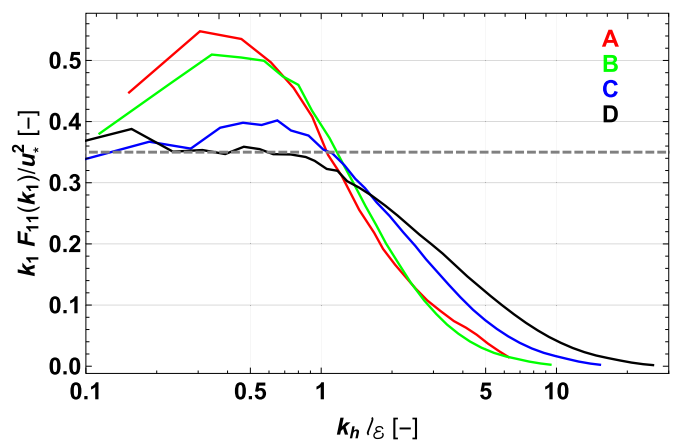

FIG. 21. Compensated spectra (left) $k_{h} E_{h}\left(k_{h}\right)$ and (right) $k_{1} F_{11}\left(k_{1}\right)$ at $z / z_{i}=0.1$ for runs A-D. The spectra have been nondimensionalized with $u_{\star}^{-2}$. The dashed gray lines indicate the plateau level. 
Figs. 19 and 20. For streamwise velocity, the negative skewness agrees with the exact result in turbulence theory (i.e., the " $-4 / 5$ " law Frisch 1995); however, the constant (i.e., 4/5) is not reproduced by our LES (not shown). Skewness is more negative for the streamwise velocity component compared to the vertical component. For all resolutions and at both heights, skewness approaches zero for large values of the horizontal separation distance $r$ for both streamwise and vertical velocity components. Velocity skewness and kurtosis show subtle mesh dependency for both streamwise and vertical velocity components at both heights. Vertical velocity reveals the largest kurtosis occurring at $z / z_{i} \sim 0.5$; where mesh dependence between runs $\mathrm{C}$ and $\mathrm{D}$ is again very small at $z / z_{i} \sim 0.5$. For large $r$, the vertical component is slightly super-Gaussian with a kurtosis just above a value of 3 , while the streamwise component at similar $r$ are below 3 (i.e., sub-Gaussian at $z / z_{i} \sim 0.1$ and closer to Gaussian at $z / z_{i} \sim 0.5$ ); Stevens et al. (2014) found similar results. Also evident in the plots are the decreasing distance with increasing resolution at which both skewness and kurtosis depart from their near-Gaussian state (i.e., Sk $\sim 0$ and $K \sim 3$ ).

Davidson et al. (2006) demonstrated that the streamwise structure functions follow a generalized logarithmic law for scales larger than the inertial range and smaller than some fraction of $z_{i}$, and showed that this is equivalent to assuming that a hierarchy of eddies exists whose kinetic energy scales as $u_{\star}^{2}$. Based on scaling arguments for higher-order moments of streamwise velocity fluctuations (Meneveau and Marusic 2013), De Silva et al. (2015) postulated that even-numbered structure functions of streamwise velocity increments scale as

$$
\left\langle\delta u_{h}(r)^{2 p}\right\rangle^{1 / p}=E_{p}^{\prime}+D_{p}^{\prime} \ln \left(\frac{r}{z}\right)
$$

for scales $z<r \ll h_{z_{i}}$, where $h_{z_{i}}$ is a fraction of the turbulent boundary layer depth $z_{i}$. $E_{p}^{\prime}$ and $D_{p}^{\prime}$ are expected to be universal functions in high-Reynolds-number turbulence; De Silva et al. (2015) finds that this might indeed be true based on various experimental datasets of very-high-Reynolds-number turbulence.

From proposing a logarithmic region via the secondorder structure function, it then immediately follows that the velocity spectra should scale like $k^{-1}$. Davidson et al. (2006) explain the difficulties in observing $k^{-1}$ spectral scaling law in one-dimensional spectra $F_{11}\left(k_{1}\right)$, and the authors list reasons similar to those mentioned in the previous section; two-dimensional ring-averaged spectra should not have the same issues. Figure 21 presents one-dimensional (right panel) and two-dimensional ring-averaged (left panel) spectra for all four simulations

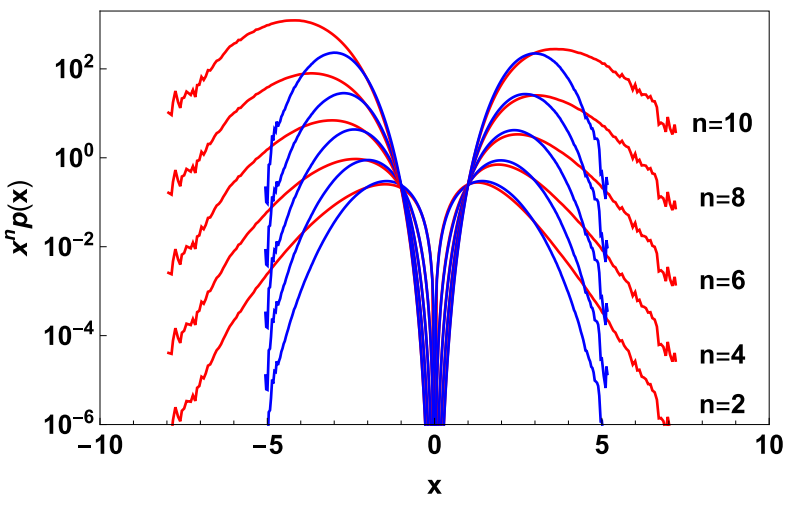

FIG. 22. The $n$th moment of the pdfs of velocity increments $p(x)$ in the streamwise direction at $z / z_{i}=0.1$ for run $\mathrm{D}$ with $x=\delta u_{h}(r) / \sigma_{\delta u_{h}(r)}$ for smallest value of $r=\Delta x$ (red lines) and largest value of $r=L_{x} / 2$ (blue lines) for $n=2,4,6,8$, and 10 .

at a height of $z / z_{i}=0.1$, a height at which it has been previously demonstrated that shear production and dissipation balance (at least for the finest-resolution simulation, run D). Pan and Chamecki (2016) showed the possibility of a more general scaling law that does not require strict balance between dissipation and production. Following Davidson and Krogstad (2014), Pan and Chamecki (2016), and Chamecki et al. (2017), we define a characteristic length scale $\ell_{\mathscr{E}}=u_{\star}^{3} / \mathscr{E}$, recognizing the importance of dissipation compared to classical surface layer scaling $\kappa z$ in characterizing the turbulent eddies in the inertial range. A comparison reveals the ratios $\kappa z / \ell_{\mathscr{E}}=\{0.58,0.73,0.84,0.93\}$ for the four simulations (runs A-D) approach a value of one with increasing resolution; a result similar to that showed in Fig. 6 where the balance between shear production and dissipation improves with increasing resolution. In Fig. 21, a very distinct plateau at $k \ell_{\mathscr{E}} \sim 1$ can be seen in both the $k_{h} E_{h}\left(k_{h}\right)$ and in $k_{1} F_{11}\left(k_{1}\right)$ spectra indicating $k^{-1}$ scaling; where run $\mathrm{D}$ scales the best and the scaling is almost nonexistent in run A. The nearly perfect scaling of $k_{1} F_{11}\left(k_{1}\right)$ for very low $k \ell_{\check{\delta}}$ is likely fortuitous compared to the narrower range observed in $k_{h} E_{h}\left(k_{h}\right)$.

We now look at the higher-order structure functions in the streamwise direction $\left\langle\delta u_{h}(r)^{n}\right\rangle$ at $z / z_{i} \sim 0.1$. Since the moments are formally calculated from

$$
\left\langle\delta u_{h}(r)^{n}\right\rangle=\int \delta u_{h}(r)^{n} p\left[\delta u_{h}(r)\right] d \delta u_{h},
$$

where $p\left[\delta u_{h}(r)\right]$ is the probability density function of increments $\delta u_{h}(r)$. Figure 22 plots the integrand for $n=2, \ldots, 10$ for the smallest and largest values of $r$. All integrands for $n=2,4$ are converged (less than 0.001 on the vertical axis) while $n=6$ is borderline and $n=8,10$ are not fully converged for the smallest value 

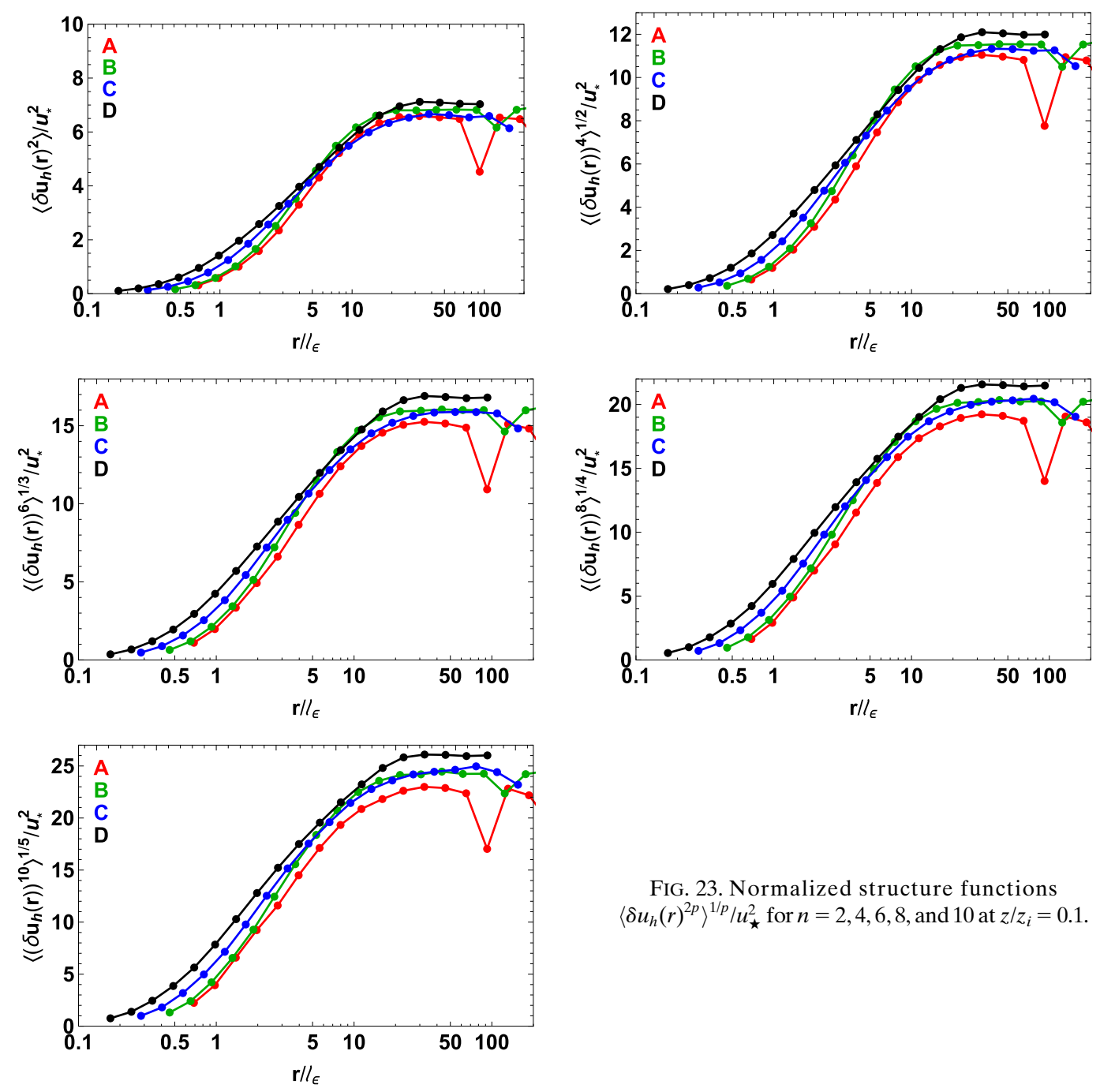

FIG. 23. Normalized structure functions $\left\langle\delta u_{h}(r)^{2 p}\right\rangle^{1 / p} / u_{\star}^{2}$ for $n=2,4,6,8$, and 10 at $z / z_{i}=0.1$.

of $r$. Though, it is important to remember that the generalized $\log$ law [Eq. (19)] is only valid for $r>z$, hence nonconvergence for values of $r$ well within the inertial range is not terribly crucial.

The findings in Davidson and Krogstad (2014), Pan and Chamecki (2016), and Chamecki et al. (2017) leads us to evaluate the following definition in our mesh sensitivity study of the streamwise structure functions:

$$
\frac{\left\langle\delta u_{h}(r)^{2 p}\right\rangle^{1 / p}}{u_{\star}^{2}}=E_{p}+D_{p} \ln \left(\frac{r}{\ell_{\mathscr{C}}}\right)
$$

for order $n=2 p$ (i.e., only considering the evenordered structure functions); the even-ordered structure functions are presented in Fig. 23. Notice that changing our length scale choice from $z$ to $\ell_{\mathscr{E}}$ implies that $E_{p} \neq E_{p}^{\prime}$ and $D_{p} \neq D_{p}^{\prime}$. All structure functions reach

an asymptotic state for $r / \ell_{\mathscr{E}} \sim 30-50$, where this state corresponds to distances that are completely decorrelated. Hence, $\left\langle\delta u_{h}(r)^{2}\right\rangle=2 \sigma_{u_{h}}^{2}$.

The logarithmic region more clearly presents itself when plotting the structure functions as function of $\ln \left(r / \ell_{\mathscr{E}}\right)$; where, Fig. 24 presents results for run D. Using an approach similar to Pan and Chamecki (2016), a least squares fit from $r=\ell_{\mathscr{E}}$ to $r=0.4 z_{i}$ shows a coefficient of determination $R^{2}>0.99$ clearly indicating the existence of a logarithmic relationship. Figure 25 shows $D_{p}$ and $E_{p}$ derived from the fit for each simulation. For $n \leq 6$ the mesh dependence for $D_{p}$ is small with stronger intermittency effects in the finer-resolution simulation (run $D$ ), in agreement the velocity skewness and kurtosis (Fig. 19). For $n=8,10$ the mesh dependence increases as do the error-bar magnitudes. A similar picture arises in the plot of $E_{p}$. Both panels in Fig. 25 include the data from De Silva et al. (2015) presented as gray dots. $D_{p}$ 


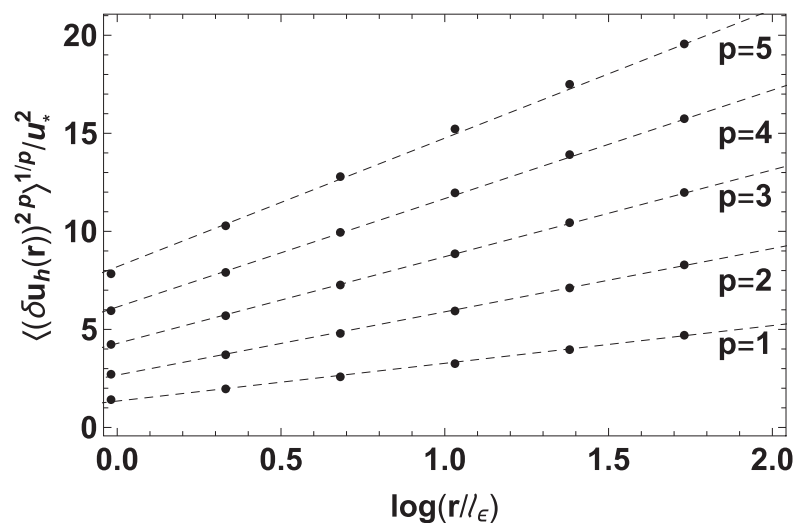

FIG. 24. Least squares fits of the generalized logarithmic law in Eq. (21) for simulation D. $R^{2}>0.99$ for all fits.

from the current simulations agrees nicely with the De Silva et al. (2015) data, while $E_{p}$ values are low in the LES data compared to the observations (i.e., the higherorder moments are suppressed). Since the high moments are mainly associated with fluctuations of the smallest scales of motion (i.e., close to the inertial range the SGS influence is here also the largest), we would therefore also expect lower values of $E_{p}$. Since $E_{p}$ depends on the chosen characteristic length scale, we also show $E_{p}$ using $z$ instead of $\ell_{\mathscr{\delta}}$ for run D (orange dots). For $n=2,4$ data using $z$ as the length scale agree well, while the LES values remain lower for higher values of $n$. Pan and Chamecki (2016) also compared their atmospheric canopy flow LES data against the data from De Silva et al. (2015) and found nice agreement for $n=2$, but did not look at higher values of $n$. Careful inspection of Pan and Chamecki's (2016) Fig. 7 could indicate some mesh dependence with similar shape to that found here (i.e., decreasing values of $D_{p}$ and increasing values of $E_{p}$ with increasing LES Reynolds number $\operatorname{Re}_{\ell}$ ). In addition, Pan and Chamecki (2016) studied meshes spanning $54 \leq \mathrm{Re}_{\ell} \leq 136$, which are notably lower than those studied here $182 \leq \mathrm{Re}_{\ell} \leq 2012$ (see Table 2).

We can also use the $k^{-1}$ scaling observed in Fig. 21 to estimate $D_{2}$, since

$$
S(k)=\gamma u_{\star}^{2} k^{-1},
$$

with $\gamma=D_{2} / 4$ in the limit where $\operatorname{Re} \rightarrow \infty$. In Eq. (22), the notation $S(k)$ represents any streamwise velocity spectrum. Using the appropriate proportionality constants predicted from their inertial range behavior, $\gamma=0.35$ for $F_{11}\left(k_{1}\right)$ and $\gamma=0.93 /[0.54 \times(55 / 18)]=0.57$ for $E_{h}\left(k_{h}\right)$, which correspond to $D_{2}=1.4$ and $D_{2}=2.2$ for the onedimensional and two-dimensional spectra, respectively. From the second-order structure function, $D_{p}=1.93 \pm$ 0.11 . Thus, ring-averaged two-dimensional spectra perform much better than one-dimensional spectra for such predictions.

The findings thus indicate that non-Gaussian statistics on scales in the $k^{-1}$ regime (i.e., scales larger than the inertial subrange), found in the LES is in both qualitative and quantitative agreement with flows at much higher Reynolds number at least up to order $n=6$. This is despite the existence of a distinct inertial range scaling at $z / z_{i} \sim 0.1$ where the analysis was carried out. Thus direct impact from the SGS model cannot be ruled out.
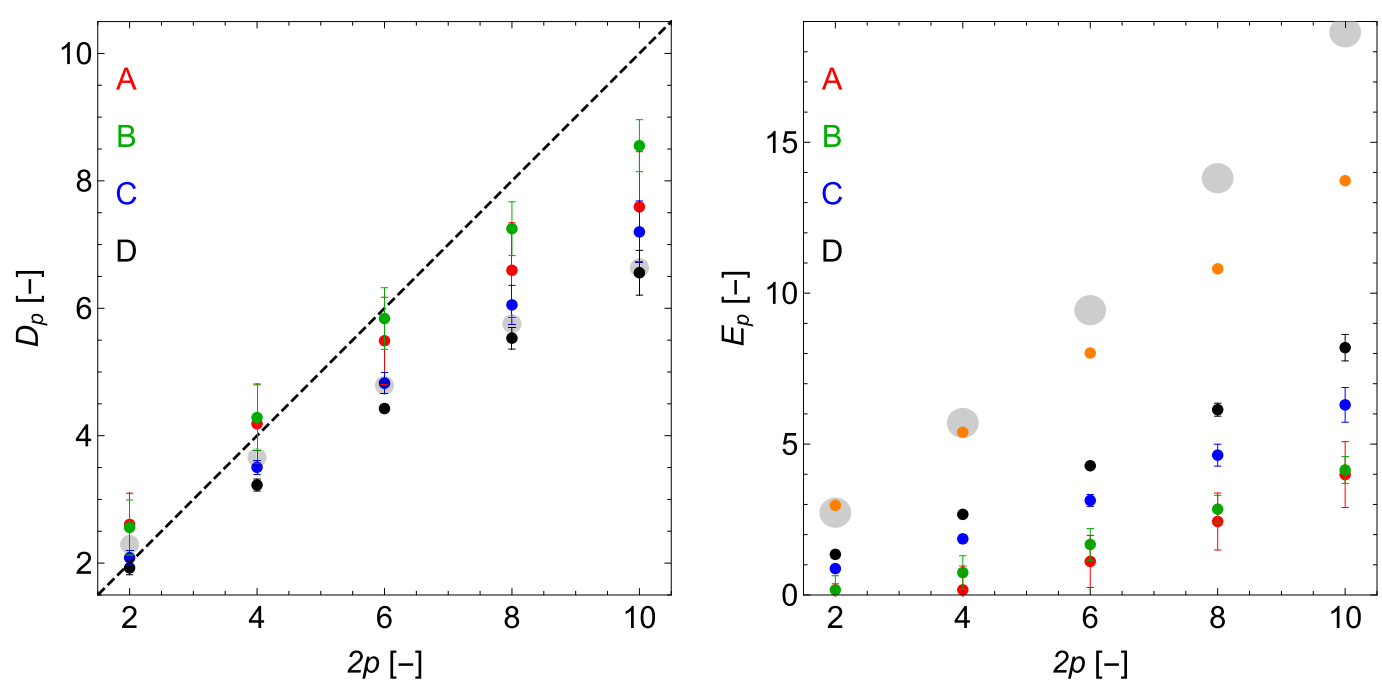

FIG. 25. Constants (left) $D_{p}$ and (right) $E_{p}$ from Eq. (21) for the four simulations. Error bars are given as the $2 \sigma$ confidence interval of the least squares fits in Fig. 24. The gray dots are the results from De Silva et al. (2015) and the orange dots are $E_{p}$ for the fine simulation (run D) using $z$ instead of $\ell_{\&}$ as the length scale. 
We however also know from Fig. 10 that the range at which the $\phi_{m}=1$ is narrow and so then is the heights at which these results can be applied.

\section{Conclusions}

A canonical neutral atmospheric boundary layer is simulated using large-eddy simulation on four different meshes. As is standard practice with LES, the simulations use a mesh-dependent subgrid-scale (SGS) parameterization that alters the partitioning between resolved and SGS motions. Hence designing metrics to assess solution convergence is not straightforward. Because of the high computational cost of threedimensional time-dependent calculations mesh studies are not typically performed when using LES for applications (e.g., within wind energy meteorology). However, our findings for a neutral atmospheric boundary layer suggest that the statistics are not fully converged with horizontal mesh spacing $\Delta \sim 2.5 \mathrm{~m}$. The mesh dependence is largest in areas of the domain where the spatial scales of turbulence are small (i.e., near the surface and at the top of the ABL in the entrainment zone). Vertical profiles of $\sqrt{\mathrm{TKE}} / S$ quantify the solution sensitivity to the mesh resolution. These show that LES indeed provide close-to-mesh independent profiles of integrated and nondimensional quantities from approximately $10 \%-$ $90 \%$ of the height of the ABL. This means that LES can directly be used even at coarser resolution if only such quantities are desired. We have, however, also shown that the resolved part of the TKE is highly dependent on the mesh resolution throughout the ABL: the contribution from SGS energy is increasing with approximately $50 \%$ each time the filter $\Delta_{f}$ is doubled. In a wind energy application context this has pros and cons: modern wind turbines are huge machines and often have hub heights in the range $120-170 \mathrm{~m}$ (i.e., they are located above the surface layer where we have shown that for example turbulence intensity is mesh independent). On the other hand, during low ABL heights the turbines might enter the entrainment layer at the top of the ABL, where processes involving buoyancy become important. To properly resolve these processes high resolution is needed-even higher than the mesh sizes utilized in this paper. If we, furthermore, want to address the dynamical loading through velocity fluctuations on the various parts on a wind turbines, such as blades and tower, accurate prediction of the spectral properties of turbulence is needed.

All of the simulations produce non-Gaussian statistics, but the scale at which the non-Gaussianity becomes significant diminishes with increasing resolution (i.e., using data from an LES performed on a coarse mesh in an application where the wind field is low-pass filtered) would predict erroneous non-Gaussian statistics. In some ways the averaging of wind turbine rotors can be seen as a low-pass filter, and there are ongoing discussions surrounding whether non-Gaussian statistics affect wind turbine operations (Milan et al. 2013; Berg et al. 2016; Schottler et al. 2017; Meneveau 2019).

The generalized log law for even-ordered structure functions in the $k^{-1}$ regime is found with meshdependent exponents matching previous studies for orders $n=2,4,6$ where convergence has been obtained. We take this as support for the maturity and size of LESs and hence the role LES deserves in applications where high accuracy and low intrinsic uncertainty are required.

Acknowledgments. JB acknowledges support from the Danish Carlsberg Foundation to a long-term visit to NCAR (2014-15). EGP and PPS acknowledge support from the National Center for Atmospheric Research, which is a major facility sponsored by the National Science Foundation under Cooperative Agreement 1852977.

\section{REFERENCES}

Abkar, M., and F. Porté-Agel, 2013: The effect of free-atmosphere stratification on boundary-layer flow and power output from very large wind farms. Energies, 6, 2338-2361, https://doi.org/ 10.3390/en6052338.

Allaerts, D., and J. Meyers, 2015: Large eddy simulation of a large wind-turbine array in a conventionally neutral atmospheric boundary layer. Phys. Fluids, 27, 065108, https://doi.org/ 10.1063/1.4922339.

— , and — 2017: Boundary-layer development and gravity waves in conventionally neutral wind farms. J. Fluid Mech., 814, 95-130, https://doi.org/10.1017/jfm.2017.11.

Ansorge, C., 2019: Scale dependence of atmospheric-surface coupling through similarity theory. Bound.-Layer Meteor., 170,127, https://doi.org/10.1007/s10546-018-0386-y.

Beare, R. J., and Coauthors, 2006: An intercomparison of large-eddy simulations of the stable boundary layer. Bound.-Layer Meteor., 118, 247-272, https://doi.org/10.1007/ s10546-004-2820-6.

Berg, J., J. Mann, and E. G. Patton, 2013: Lidar-observed stress vectors and veer in the atmospheric boundary layer. J. Atmos. Oceanic Technol., 30, 1961-1969, https://doi.org/ 10.1175/JTECH-D-12-00266.1.

_ - A. Natarajan, J. Mann, and E. G. Patton, 2016: Gaussian vs non-Gaussian turbulence: Impact on wind turbine loads. Wind Energy, 19, 1975-1989, https://doi.org/10.1002/we.1963.

Bou-Zeid, E., 2015: Challenging the large eddy simulation technique with advanced a posteriori tests. J. Fluid Mech., 764, 1-4, https://doi.org/10.1017/jfm.2014.616.

, C. Meneveau, and M. Parlange, 2005: A scale-dependent Lagrangian dynamic model for large eddy simulation of complex turbulent flows. Phys. Fluids, 17, 025105, https:// doi.org/10.1063/1.1839152. 
- N. Nercauteren, M. B. Parlange, and C. Meneveau, 2008: Scale dependence of subgrid-scale model coefficients: An a priori study. Phys. Fluids, 20, 115106, https://doi.org/10.1063/ 1.2992192 .

Brasseur, J. G., and T. Wei, 2010: Designing large-eddy simulation of the turbulent boundary layer to capture law-of-the-wall scaling. Phys. Fluids, 22, 021303, https://doi.org/10.1063/ 1.3319073 .

Calaf, M., C. Meneveau, and J. Meyers, 2010: Large eddy simulation study of fully developed wind-turbine array boundary layers. Phys. Fluids, 22, 015110, https://doi.org/10.1063/ 1.3291077 .

Chamecki, M., N. L. Dias, S. T. Salesky, and Y. Pan, 2017: Scaling laws for the longitudinal structure function in the atmospheric surface layer. J. Atmos. Sci., 74, 1127-1147, https://doi.org/ 10.1175/JAS-D-16-0228.1.

Churchfield, M. J., S. Lee, J. Michalakes, and P. J. Moriarty, 2012: A numerical study of the effects of atmospheric and wake turbulence on wind turbine dynamics. J. Turbul., 13, N14, https://doi.org/10.1080/14685248.2012.668191.

Davidson, P. A., and P. A. Krogstad, 2014: A universal scaling for low-order structure functions in the log-law region of smoothand rough-wall boundary layers. J. Fluid Mech., 752, 140-156, https://doi.org/10.1017/jfm.2014.286.

— T. B. Nickels, and P. A. Krogstad, 2006: The logarithmic structure function law in wall-layer turbulence. J. Fluid Mech., 550, 51-60, https://doi.org/10.1017/S0022112005008001.

De Silva, C. M., I. Marusic, J. D. Woodcock, and C. Meneveau, 2015: Scaling of second- and higher-order structure functions in turbulent boundary layers. J. Fluid Mech., 769, 654-686, https://doi.org/10.1017/jfm.2015.122.

Deardorff, J. W., 1970: A numerical study of three-dimensional turbulent channel flow at large Reynolds numbers. J. Fluid Mech., 41, 453-480, https://doi.org/10.1017/S0022112070000691. , 1972: Numerical investigation of neutral and unstable planetary boundary layers. J. Atmos. Sci., 29, 91-115, https://doi.org/ 10.1175/1520-0469(1972)029<0091:NIONAU>2.0.CO;2.

- 1980: Stratocumulus-capped mixed layers derived from a three-dimensional model. Bound.-Layer Meteor., 18, 495-527, https://doi.org/10.1007/BF00119502.

Dougherty, J., 1961: The anisotropy of turbulence at the meteor level. J. Atmos. Terr. Phys., 21, 210-213, https://doi.org/ 10.1016/0021-9169(61)90116-7.

Ercolani, G., C. Gorlé, C. Corbari, and M. Mancini, 2017: RAMS sensitivity to grid spacing and grid aspect ratio in large-eddy simulations of the dry neutral atmospheric boundary layer. Comput. Fluids, 146, 59-73, https://doi.org/ 10.1016/j.compfluid.2017.01.010.

Frisch, U., 1995: Turbulence-The Legacy of A. N. Kolmogorov. Cambridge University Press, 296 pp.

International Electrotechnical Commission, 2005: Wind turbines-Part 1: Design requirements. Tech. Doc. IEC 61400-1, International Electrotechnical Commission, $70 \mathrm{pp}$.

Jiang, Q., S. Wang, and P. Sullivan, 2018: Large-eddy simulation study of log laws in a neutral Ekman boundary layer. J. Atmos. Sci., 75, 1873-1889, https://doi.org/10.1175/JAS-D-17-0153.1.

Jiménez, J., 2012: Cascades in wall-bounded turbulence. Annu. Rev. Fluid Mech., 44, 27-45, https://doi.org/10.1146/annurevfluid-120710-101039.

Jonker, H. J., M. Van Reeuwijk, P. P. Sullivan, and E. G. Patton, 2013: On the scaling of shear-driven entrainment: A DNS study. J. Fluid Mech., 732, 150-165, https://doi.org/10.1017/ jfm.2013.394.
Kelly, M., and J. C. Wyngaard, 2006: Two-dimensional spectra in the atmospheric boundary layer. J. Atmos. Sci., 63, 3066-3070, https://doi.org/10.1175/JAS3769.1.

Klemp, J. B., and D. R. Durran, 1983: An upper boundary condition permitting internal gravity wave radiation in numerical mesoscale models. Mon. Wea. Rev., 111, 430-444, https://doi.org/ 10.1175/1520-0493(1983)111<0430:AUBCPI>2.0.CO;2.

Kosović, B., and J. A. Curry, 2000: A large eddy simulation study of a quasi-steady, stably stratified atmospheric boundary layer. J. Atmos. Sci., 57, 1052-1068, https://doi.org/10.1175/15200469(2000)057<1052:ALESSO > 2.0.CO;2.

Lilly, D., 1967: The representation of small-scale turbulence in numerical simulation experiments. Proc. 10th IBM Scientific Computing Symp. on Environmental Sciences, Yorktown Heights, NY, IBM Thomas J. Watson Research Center, 195-210.

Lin, C., J. C. McWilliams, C. Moeng, and P. P. Sullivan, 1996: Coherent structures and dynamics in a neutrally stratified planetary boundary layer flow. Phys. Fluids, 8, 2626-2639, https://doi.org/10.1063/1.869048.

Meneveau, C., 2019: Big wind power: Seven questions for turbulence research. J. Turbul., 20, 2-20, https://doi.org/10.1080/ 14685248.2019.1584664.

_ large-eddy simulation. Annu. Rev. Fluid Mech., 32, 1-32, https://doi.org/10.1146/annurev.fluid.32.1.1.

_ order moments in turbulent boundary layers. J. Fluid Mech., 719, R1-R11, https://doi.org/10.1017/jfm.2013.61.

Milan, P., M. Wächter, and J. Peinke, 2013: Turbulent character of wind energy. Phys. Rev. Lett., 110, 138701, https://doi.org/ 10.1103/PhysRevLett.110.138701.

Moeng, C.-H., 1984: A large-eddy-simulation model for the study of planetary boundary-layer turbulence. J. Atmos. Sci., $\mathbf{4 1}$, 2052-2062, https://doi.org/10.1175/1520-0469(1984)041<2052: ALESMF $>2.0 . \mathrm{CO} ; 2$.

— ulations of the convective boundary layer. J. Atmos. Sci., $\mathbf{4 5}$, 3573-3587, https://doi.org/10.1175/1520-0469(1988)045<3573: SAOLES $>2.0 . \mathrm{CO} ; 2$.

— , and P. P. Sullivan, 1994: A comparison of shear- and bouyancy-driven planetary boundary layer flows. J. Atmos. Sci., 51, 999-1022, https://doi.org/10.1175/1520-0469(1994) 051<0999:ACOSAB > 2.0.CO;2.

Moin, P., and K. Mahesh, 1998: Direct numerical simulation: A tool in turbulence research. Annu. Rev. Fluid Mech., 30, 539-578, https://doi.org/10.1146/annurev.fluid.30.1.539.

Orszag, S., 1971: On the elimination of aliasing in finite-difference schemes by filtering high-wavenumber components. J. Atmos. Sci., 28, 1074, https://doi.org/10.1175/1520-0469(1971)028<1074: OTEOAI $>2.0 . \mathrm{CO} ; 2$.

Otte, M. J., and J. C. Wyngaard, 2001: Stably stratified interfaciallayer turbulence from large-eddy simulation. J. Atmos. Sci., 58, 3424-3442, https://doi.org/10.1175/1520-0469(2001)058<3424: SSILTF $>2.0 . C O ; 2$.

Ozmidov, R. V., 1965: On the turbulent exchange in a stably stratified ocean. Izv. Acad. Sci. USSR, Atmos. Oceanic Phys., 1, 853-860.

Pan, Y., and M. Chamecki, 2016: A scaling law for the shearproduction range of second-order structure functions. J. Fluid Mech., 801, 459-474, https://doi.org/10.1017/jfm.2016.427.

Panofsky, H. A., and J. A. Dutton, 1984: Atmospheric Turbulence. John Wiley and Sons, 397 pp. 
Pedersen, J., S.-E. Gryning, and M. Kelly, 2014: On the structure and adjustment of inversion-capped neutral atmospheric boundary-layer flows: Large-eddy simulation study. Bound. Layer Meteor., 153, 43-62, https://doi.org/10.1007/s10546014-9937-z.

Peltier, L. J., J. C. Wyngaard, S. Khanna, and J. G. Brasseur, 1996: Spectra in the unstable surface layer. J. Atmos. Sci., 53, 49-61, https://doi.org/10.1175/1520-0469(1996)053<0049: SITUSL $>2.0 . \mathrm{CO} ; 2$.

Pollard, R. T., P. B. Rhines, and R. O. R. Y. Thompson, 1973: The deepening of the wind-mixed layer. Geophys. Fluid Dyn., $\mathbf{4}$ 381-404, https://doi.org/10.1080/03091927208236105.

Pope, S. B., 2000: Turbulent Flows. Cambridge University Press, $770 \mathrm{pp}$.

Porté-Agel, F., C. Meneveau, and M. Parlange, 2000: A scaledependent dynamic model for large-eddy simulation: Application to a neutral atmospheric boundary layer. J. Fluid Mech., 415, 261-284, https://doi.org/10.1017/S0022112000008776.

Schottler, J., N. Reinke, A. Hölling, J. Whale, J. Peinke, and M. Hölling, 2017: On the impact of non-Gaussian wind statistics on wind turbines-An experimental approach. Wind Energy Sci., 2, 1-13, https://doi.org/10.5194/wes-2-1-2017.

Sørensen, J. N., R. F. Mikkelsen, D. S. Henningson, S. Ivanell, S. Sarmast, and S. J. Andersen, 2015: Simulation of wind turbine wakes using the actuator line technique. Philos. Trans. Roy. Soc., 373, 20140071, https://doi.org/10.1098/rsta.2014.0071.

Sreenivasan, K. R., 1995: On the universality of the Kolmogorov constant. Phys. Fluids, 7, 2778-2784, https://doi.org/10.1063/ 1.868656 .
Stevens, R. J., M. Wilczek, and C. Meneveau, 2014: Large-eddy simulation study of the logarithmic law for second- and higherorder moments in turbulent wall-bounded flow. J. Fluid Mech., 757, 888-907, https://doi.org/10.1017/jfm.2014.510.

Sullivan, P. P., and E. G. Patton, 2011: The effect of mesh resolution on convective boundary layer statistics and structures generated by large-eddy simulation. J. Atmos. Sci., 68, 23952415, https://doi.org/10.1175/JAS-D-10-05010.1.

_ J. C. McWilliams, and C.-H. Moeng, 1994: A subgrid-scale model for large-eddy simulation of planetary boundary-layer flows. Bound.-Layer Meteor., 71, 247-276, https://doi.org/ 10.1007/BF00713741.

—, C.-H. Moeng, B. Stevens, D. H. Lenschow, and S. D. Mayor, 1998: Structure of the entrainment zone capping the convective atmospheric boundary layer. J. Atmos. Sci., 55, 3042-3064, https:// doi.org/10.1175/1520-0469(1998)055<3042:SOTEZC > 2.0.CO;2. , J. C. Weil, E. G. Patton, H. J. J. Jonker, and D. V. Mironov, 2016: Turbulent winds and temperature fronts in large-eddy simulations of the stable atmospheric boundary layer. J. Atmos. Sci., 73, 1815-1840, https://doi.org/10.1175/ JAS-D-15-0339.1.

Taylor, G. I., 1938: The spectrum of turbulence. Proc. Roy. Soc. London A, 164, 476-490, https://doi.org/10.1098/rspa.1938.0032.

Wyngaard, J. C., 2010: Turbulence in the Atmosphere. Cambridge University Press, 393 pp.

Zilitinkevich, S., I. Esau, and A. Baklanov, 2007: Further comments on the equilibrium height of neutral and stable planetary boundary layers. Quart. J. Roy. Meteor. Soc., 133, 265-271, https://doi.org/10.1002/QJ.27. 